\title{
Geometry and Space-Time Extent of Pion Emission Region at FCC Energies
}

\author{
V. A. Okorokov \\ National Research Nuclear University MEPhI (Moscow Engineering Physics Institute), Kashirskoe Shosse 31, Moscow 115409, Russia \\ Correspondence should be addressed to V. A. Okorokov; vaokorokov@mephi.ru
}

Received 10 August 2016; Revised 1 October 2016; Accepted 20 October 2016

Academic Editor: Burak Bilki

Copyright ( 2016 V. A. Okorokov. This is an open access article distributed under the Creative Commons Attribution License, which permits unrestricted use, distribution, and reproduction in any medium, provided the original work is properly cited. The publication of this article was funded by $\mathrm{SCOAP}^{3}$.

\begin{abstract}
The energy dependence is investigated for a wide set of space-time characteristics derived from Bose-Einstein correlations of secondary pion pairs produced in proton-proton and nucleus-nucleus interactions. Analytic functions suggested for smooth approximations of the energy dependence of emission region parameters demonstrate reasonable agreement with all available experimental results for proton-proton collisions while the approximations correspond to most of experimental data for nucleusnucleus collisions at energies above $5 \mathrm{GeV}$. Estimations for a wide set of space-time quantities are obtained for energies for the Future Circular Collider (FCC) project based on the smooth approximations. The space particle densities at freeze-out are derived also from estimations for the volume of the emission region and for total multiplicity at FCC energies. Estimations for charged particle density and its critical value allow the possibility of lasing behavior for secondary pions in nucleus-nucleus collisions at FCC energy. The mathematical formalism is presented for study of the peak shape of correlation function for general case of central-symmetrical Lévy-Feldheim distribution.
\end{abstract}

\section{Introduction}

When two energetic particles or nuclei collide, some matter is created in finite space-time volume. This matter volume, often called "fireball," emits particles and space-time extent of the fireball is of fundamental interest for understanding of both the multiparticle production dynamics and the evolution of early Universe. One of the collective effects, namely, particle correlations at low relative momentum, represents a unique tool and sensitive probe of the size and the shape of the fireball at the last stage of its evolution (colorless particle emission region). The space-time geometry of particle source can be determined by using a method of interferometry based on the fundamental relation between spin and statistics. The production of identical bosons that are close together in phase space is enhanced by the presence of quantum statistical effect on Bose-Einstein correlations (BEC). The strength and form of the correlation reflect the space-time structure of the source [1]. The most of the secondary particles produced in the strong interactions are pions. Thus in the paper correlations between two identical bosons called BEC are studied for secondary charged pions produced in various strong interaction processes (in these reactions the BEC are often called HBT correlations due to analogy with HanburyBrown and Twiss effect $[2,3]$ used in radio astronomy to measure the angular sizes of stellar objects).

The international project called Future Circular Collider (FCC) is mostly aimed at hadron collider with a centreof-mass energy $\sqrt{s_{\mathrm{pp}}}=100 \mathrm{TeV}$ for $p+p$ collisions in a new $100 \mathrm{~km}$ tunnel of the CERN accelerator complex and detailed characteristics of various beams for FCC can be found elsewhere [4]. For heavy ion collisions the relation $\sqrt{s_{\mathrm{NN}}}=\sqrt{s_{\mathrm{pp}} \times\left(Z_{1} Z_{2} / A_{1} A_{2}\right)}$ gives the energy in centre-ofmass per nucleon-nucleon collision of $\sqrt{s_{\mathrm{NN}}}=39 \mathrm{TeV}$ for $\mathrm{Pb}$ $+\mathrm{Pb}(Z=82, A=208)$ and $63 \mathrm{TeV}$ for $p+\mathrm{Pb}$ collisions [5-7]. This project provides a unique opportunity to probe quantum chromodynamics (QCD) in the new energy regime [8]. One of the most distinguishing features of QCD is the mechanism of color confinement, the physics of which are not fully understood, due, in part, to its theoretical intractability [9]. The confinement mechanism has a physical scale of 
the order of the proton radius and is especially important at low momentum. Therefore study of source geometry in new energy domain with help of BEC seems important for better understanding of both the equation of state (EOS) of strongly interacting matter and general dynamic features of soft processes. The peak of two-particle Bose-Einstein correlation function (CF) contains the unique experimental information about particle source at freeze-out. The peak shape carries information, in particular, about the possible complex highly irregular geometry of the source $[10,11]$, the $U_{A}(1)$ symmetry restoration in high energy heavy ion reactions [12], and so forth. Therefore the development of a general formalism for detailed shape analysis of the peak in BEC CF is relevant for future high-statistics studies at FCC. It should be stressed that on the one hand the BEC leads also to Bose-Einstein condensates responsible for laser, superfluids, and superconductors [1]. On the other hand the pion multiplicity at midrapidity $\left.(d N / d \eta)\right|_{\eta=0}$ is larger than $10^{3}$ in heavy ion collisions in the TeV-energy domain, in particular, at $\left.\mathrm{FCC}(d N / d \eta)\right|_{\eta=0} \simeq 3600$ in $\mathrm{Pb}+\mathrm{Pb}$ collisions at $\sqrt{s_{\mathrm{NN}}}=39 \mathrm{TeV}[5,6]$. Therefore the number of pions in a unit value of phase space may be large enough that these bosons condense into the same quantum state and a pion laser could be created $[13,14]$. Thus the paper is focused on the study of azimuthally integrated BEC of secondary charged pions produced in strong interactions, especially, on the space-time extent of pion emission region and the possible novel features of multiparticle production mechanism (pion laser) at FCC energies. Also the general formalism is suggested for study of shape of correlation peak in detail.

The paper is organized as follows. In Section 2, definitions of two-particle CF and BEC parameters are described. Section 3 devotes discussion of energy dependence of pion source extent in $p+p$ and $\mathrm{A}+\mathrm{A}$ collisions, predictions for wide set of space-time characteristics for pion source in various collisions at FCC energies. The possibility for pion laser in strong interaction processes at FCC energies is considered in Section 4. In Section 5, the generalized parametrization of $3 \mathrm{D}$ CF is introduced with help of expansion of central-symmetrical Lévy-Feldheim distribution. Some final remarks are presented in Section 6.

\section{Method and Variables}

The BEC effect is observed as an enhancement in the twoparticle CF at low values of some difference constructed from 4 momentum $p_{i}, i=1,2$, or its components of the entering particles, $C_{2}\left(p_{1}, p_{2}\right)=\rho\left(p_{1}, p_{2}\right) / \rho_{\text {ref }}\left(p_{1}, p_{2}\right)$, where $\rho$ is the two-particle density function and $\rho_{\text {ref }}$ is a reference twoparticle density function that by construction is expected to include no BEC. Recent study [20] shows that BEC 1D experimental data samples are not enough for study of energy dependence of source parameters in proton-nucleus and nucleus-nucleus collisions. Therefore the present paper is focused on the $3 \mathrm{D}$ analysis of BEC in strong interaction processes.
In general phenomenological parametrization of $\mathrm{CF}$ with taking into account different forms of corrections on Coulomb final state interaction (FSI) can be written as follows [11]:

$$
\begin{aligned}
C_{2,(m)}^{\mathrm{ph}}(q, K) & =\zeta P_{\text {coul }}^{(m)}(q)\left[\zeta^{-1}+\mathbf{K}_{2}^{\mathrm{ph}}(\mathbf{A})\right], \\
\mathbf{K}_{2}^{\mathrm{ph}}(\mathbf{A}) & =C_{2,(m)}^{\mathrm{ph}}(q, K)-1,
\end{aligned}
$$

where $\mathbf{K}_{2}$ is the cumulant correlation function (cCF), $\zeta=\lambda$ at $m=1,2$, and $\zeta=1$ at $m=3$ while $m=1$ corresponds to the standard Coulomb correction, $m=2$ corresponds to the dilution procedure, and $m=3$ corresponds to the BowlerSinyukov correction, $q \equiv\left(q^{0}, \vec{q}\right)=p_{1}-p_{2}$ is the relative 4-momentum, $K \equiv\left(K^{0}, \vec{K}\right)=\left(p_{1}+p_{2}\right) / 2$ is the average 4-momentum of particles in pair (pair 4-momentum), $\mathbf{A} \equiv$ $\vec{q} \mathbf{R}^{2} \vec{q}^{T}$ and $\mathbf{R}^{2}$ are the matrices $3 \times 3$, and $\vec{q}^{T}$ is transposed vector $\vec{q}, \forall i, j=1-3: R_{i j}^{2}=R_{j i}^{2}$ and $R_{i i}^{2} \equiv R_{i}^{2}$, where $R_{i}=R_{i}(K)$ are parameters characterized the linear scales of the region of homogeneity $[21,22]$; the products are taken on space components of vectors, $\lambda(K)=\mathbf{K}_{2}(0, K), 0 \leq$ $\lambda \leq 1$, is the parameter which characterizes the strength of correlations called also chaoticity. Different types of Coulomb correction for two-pion correlations are compared in [11]. The space component of pair 4-momentum $(\vec{K})$ is decomposed on longitudinal $k_{\|}=\left(p_{\|, 1}+p_{\|, 2}\right) / 2$ and transverse $\vec{k}_{\perp}=$ $\left(\vec{p}_{\perp, 1}+\vec{p}_{\perp, 2}\right) / 2$ parts of pair momentum. In the paper the decomposition of Pratt-Bertsch $[23,24]$ is used for $\vec{q}$ as well as the longitudinal comoving system (LCMS) frame. The parametrization of $\mathbf{K}_{2}^{\mathrm{ph}}(\mathbf{A})$ depends on type of distribution which was chosen for emission region [11]. For instance, the lowest order $\mathrm{CCF}$ can be written as

$$
\mathbf{K}_{2, G}^{\mathrm{ph}, 0}(\mathbf{A})=\exp \left(-\sum_{i, j=1}^{3} q_{i} R_{i j}^{2} q_{j}\right),
$$

for specific case of Gaussian distribution which is one of the most used ones in BEC study. As known the study of $\mathrm{BEC}$ allows the estimation of space-time extent for region of homogeneity which is only some part of whole source. Therefore the BEC parameters $\forall i=1-3: R_{i}(K)$ are smaller a priori than corresponding scales of whole emission region and consequently the experimental BEC dimensions $R_{i}(K)$ can be considered as low boundary for corresponding true linear scales of source

$$
R_{i}(K)=\inf R_{i}^{\mathrm{tr}}(K), \quad \forall i=1-3 .
$$

For this reason the BEC parameters $\forall i=1-3: R_{i}(K)$ are called BEC radii and it is assumed that correlation analysis for pairs of identical particles with low $\left\langle\vec{k}_{\perp}\right\rangle$ provides $R_{i}$ which are adequate experimental estimations for space-time extent of whole emission region within the simplest approach at least. It should be noted that azimuthally integrated BEC analysis allows rougher estimations for space-time scales of whole source with increase of collision energy because more intensive collective expansions reduce the sizes of the region of homogeneity more significantly at higher energies. Thus in 
the present paper $R_{i}, i=1-3$, are considered as source BEC radii with taking into account relation (3) and influence of collective flows on the quality of this approximation.

In the 3D case and the Pratt-Bertsch coordinate system the space-time extents of the region of homogeneity or, with taking into account the discussion above, whole source is described by the following dimensions: $R_{l}$ is the source size along the beam axis, $R_{o}$ is extent along $\vec{k}_{\perp}$, and $R_{s}$ is the source size along the axis perpendicular to those two. Then one can define the geometric mean $\mathrm{BEC}$ radius

$$
R_{m}^{3}=\prod_{i=s, o, l} R_{i}
$$

as well as the difference

$$
\delta \equiv R_{o}^{2}-R_{s}^{2}
$$

which is an important observable especially for some specific cases of $1 \mathrm{D}$ hydrodynamics (static, nonflowing source) due to its relation with particle emission duration $\delta \approx \beta_{\perp}^{2}(\Delta \tau)^{2}$ $[25,26]$, where $\beta_{\perp}=k_{\perp} / m_{\perp}$ is the transverse velocity of pair of particles with mass $m, m_{\perp}^{2}=k_{\perp}^{2}+m^{2}$. Here the scaled geometric mean BEC radius is defined as follows: $R_{m}^{n}=R_{m} / R_{A}$ in accordance with approach suggested in [11, 15], where $\left\langle R_{A}\right\rangle=\left(R_{A_{1}}+R_{A_{2}}\right) / 2$ is the mean radius for beam nuclei, $R_{A}=r_{0} A^{1 / 3}$ is radius of spherically symmetric nucleus, and $r_{0}=(1.25 \pm 0.05) \mathrm{fm}[27,28]$. The volume of source can be written as follows:

$$
\begin{aligned}
& V=(2 \pi)^{3 / 2} R_{s}^{2} R_{l}, \\
& V=\frac{4 \pi R_{m}^{3}}{3}
\end{aligned}
$$

where the first case is the standard relation for BEC while the second case corresponds to the simplest approach of spherically symmetric source and it can be useful for future study of pion laser. Thus in the paper the following set of main BEC observables $\mathscr{G}_{1} \equiv\left\{\mathscr{G}_{1}^{i}\right\}_{i=1}^{4}=\left\{\lambda, R_{s}, R_{o}, R_{l}\right\}$ is under consideration as well as the set of important additional observables which can be calculated with help of BEC radii $\mathscr{G}_{2} \equiv\left\{\mathscr{G}_{2}^{j}\right\}_{j=1}^{4}=\left\{R_{o} / R_{s}, R_{m}, \delta, V\right\}$. The set of parameters $\mathscr{G}_{1}$ characterizes the chaoticity of source and its 4-dimensional geometry at freeze-out stage completely.

\section{Space-Time Extent of Pion Source}

In this study experimental BEC data sets are from [29] for $p+p$ and from [15] for $\mathrm{A}+\mathrm{A}$ collisions.

Dependencies of BEC parameters $\mathscr{G}_{1}^{i}\left(\sqrt{s_{\mathrm{NN}}}\right), i=1-4$, for $p+p$ high energy collisions are shown in Figures $1(\mathrm{a})-1(\mathrm{~d})$, respectively. As seen for energy range from Relativistic Heavy Ion Collider (RHIC) to the Large Hadron Collider (LHC) the experimental $\lambda\left(\sqrt{s_{\mathrm{NN}}}\right)$ is close to the constant (Figure 1(a)) while some decrease is observed for experimental $\lambda\left(\sqrt{s_{\mathrm{NN}}}\right)$ deduced from 1D two-pion BEC analyses [30]. The BEC radii increase with collision energy (Figures $1(\mathrm{~b})-1(\mathrm{~d})$ ) more significantly in transverse plane with respect of the beam direction compared to that for longitudinal direction. Taking into account the view of experimental $\mathscr{G}_{1}^{i}\left(\sqrt{s_{\mathrm{NN}}}\right), i=1-4$, in $p+p$ as well as the detailed study of energy dependence of azimuthally integrated main BEC parameters $\mathscr{G}_{1}^{i}, i=1-4$, for charged pions in nucleus-nucleus interactions [15] the following function is used:

$$
f(\varepsilon)=a_{1}\left[1+a_{2}(\ln \varepsilon)^{a_{3}}\right],
$$

for smooth approximation of experimental dependencies $\mathscr{G}_{1}^{i}\left(\sqrt{s_{\mathrm{pp}}}\right), i=1-4$, in $p+p$ interactions. Here $\varepsilon \equiv s_{\mathrm{pp}} / s_{0}$ for $p+p$ or $\varepsilon \equiv s_{\mathrm{NN}} / s_{0}$ for $\mathrm{A}+\mathrm{A}$ reactions, where $s_{0}=$ $1 \mathrm{GeV}^{2}$. The very limited ensemble of experimental points from 3D Gaussian analyses of $p+p$ does not allow the fit by (7) with all parameters $\forall i=1-3: a_{i}$ to be free. In general the various quantities from the set $\mathscr{G}_{1}$ can show the different behavior as function of $\ln \varepsilon$ [15]. Thus the following two views of (7) are used for approximation of experimental points: (i.1) function (7) at fixed value of $a_{3}$ which is defined by method of sequential approximations and (i.2) the specific case of (7) at $a_{3}=1.0$. Only statistical uncertainties are available for strength of correlations $\lambda$ while for each of the BEC radii $\left\{\mathscr{G}_{1}^{i}\right\}_{i=2}^{4}$ fits are made for both the statistical and total errors, where total errors of experimental points include available clear indicated systematic errors added in quadrature to statistical ones. The numerical values of fit parameters are presented in Table 1, where the second line for chaoticity parameter $\lambda$ corresponds to the simplest fit by constant and for each of the BEC radii to the approximation by specific case of (7). Approximation curves are shown in Figure 1(a) by solid line for specific case of (7) and by dashed line for fit by constant. Figures 1(b)-1(d) show the fit results for BEC radii by solid lines for approach (i.1) and by dashed lines for specific case (i.2) with taking into account the statistical errors of experimental points.

In difference with $\mathrm{A}+\mathrm{A}$ collisions [15] function (7) for both choices of $a_{3}$ agrees with experimental $p+p$ data quantitatively and provides reasonable fit qualities even with statistical errors for all BEC parameters from the set $\mathscr{G}_{1}$ with exception of $R_{s}$. In the last case one can only conclude that fit curve is similar to the general trend of experimental points (Figure 1(b)) due to poor fit quality. Account for total errors allows statistically acceptable fit qualities for all main BEC parameters in both approaches (i.1) and (i.2). Furthermore $a_{2}$ is equal to zero within errors for longitudinal BEC radius for (i.2) and consequently $R_{l}\left(\sqrt{s_{\mathrm{pp}}}\right)$ can be described by constant with $a_{1}=(1.57 \pm 0.13) \mathrm{fm}$ and $\chi^{2} /$ n.d.f. $=0.42 / 2$ in the case of the accounting for total errors. For energy range from RHIC to the LHC fit curves for approaches (i.1) and (i.2) are close to each other for $\mathscr{G}_{1}^{i}, i=2-4$, especially for radii $R_{o}$ (Figure $1(\mathrm{c})$ ) and $R_{l}$ (Figure $1(\mathrm{~d})$ ). Nevertheless the fit within approach (i.1) for $R_{o}$ only confirms $R_{o} \propto$ $\ln \varepsilon$ for statistical errors but other BEC radii show faster increasing with $\sqrt{s_{\mathrm{pp}}}$ especially $R_{l}$ (Table 1 ). The growth of $R_{s}$ approaches the linear behavior in $\ln \varepsilon$ for accounting for total errors but longitudinal radius $R_{l}$ preserves much faster growth with energy increase in this case too. As a consequence the method of sequential approximations for 


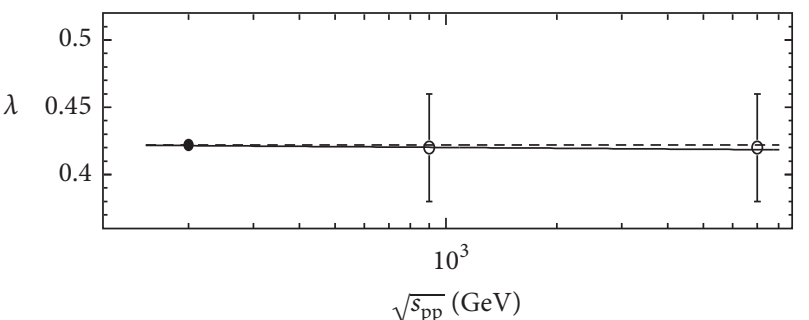

- STAR

- ALICE

(a)

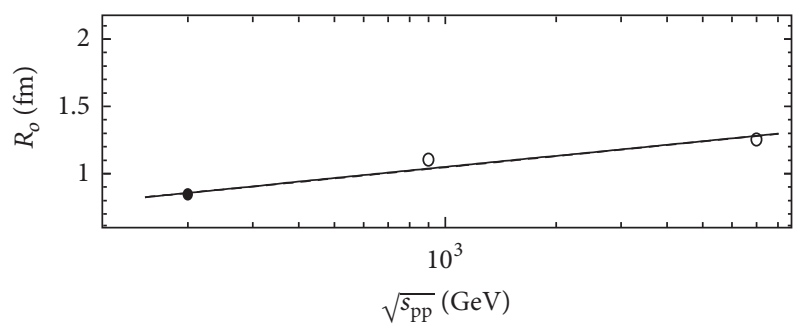

- star

- ALICE

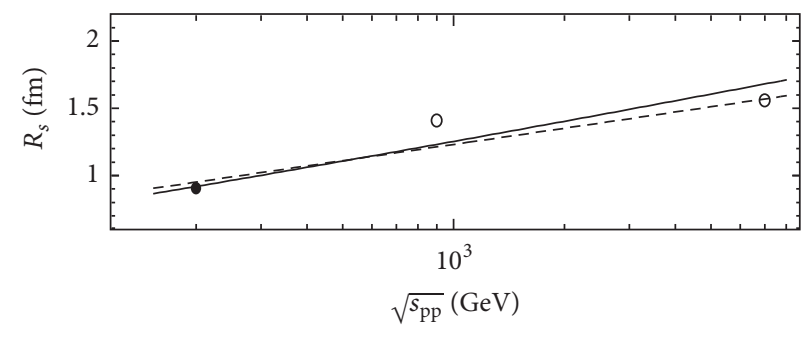

- STAR

- ALICE

(b)

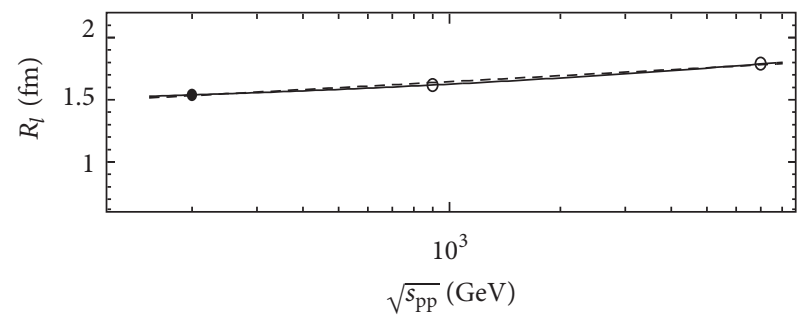

- star

- ALICE

(c)

(d)

FIGURE 1: Energy dependence of the main BEC parameters $\mathscr{G}_{1}^{i}, i=1-4$, obtained for 3D Gaussian analyses for $p+p$ collisions at midrapidity and low $\left\langle k_{T}\right\rangle \simeq 0.2 \mathrm{GeV} / c$. Errors for experimental points are statistical only. For strength of correlations (a) fit by specific case of (7) at $a_{3}=1.0$ is shown by solid line, and the dashed line corresponds to the fit by constant. For BEC radii (b-d) approximations by function (7) are shown by solid lines for case (i.1), and dashed lines present the results for linear logarithmic function in case (i.2).

TABLE 1: Values of fit parameters for approximation of $p+p$ data.

\begin{tabular}{|c|c|c|c|c|c|c|c|c|}
\hline \multirow{2}{*}{$\begin{array}{l}\text { BEC } \\
\text { parameter }\end{array}$} & \multicolumn{4}{|c|}{ Fit with statistical errors } & \multicolumn{4}{|c|}{ Fit with total errors } \\
\hline & $a_{1}$ & $a_{2}$ & $a_{3}$ & $\chi^{2} /$ n.d.f. & $a_{1}$ & $a_{2}$ & $a_{3}$ & $\chi^{2} /$ n.d.f. \\
\hline \multirow{2}{*}{$\lambda$} & $0.43 \pm 0.06$ & $-0.001 \pm 0.012$ & 1.0 & $7.0 \times 10^{-4} / 1$ & - & - & - & - \\
\hline & $0.422 \pm 0.004$ & - & - & $4.9 \times 10^{-4} / 1$ & - & - & - & - \\
\hline \multirow{2}{*}{$R_{s}$} & $(6.8 \pm 1.0) \times 10^{-3}$ & $7.57 \pm 0.24$ & 1.21 & $47.7 / 1$ & $(8.0 \pm 1.2) \times 10^{-3}$ & $7.3 \pm 1.0$ & 1.16 & $0.57 / 1$ \\
\hline & $(2.5 \pm 0.7) \times 10^{-3}$ & $35 \pm 7$ & 1.0 & $62.6 / 1$ & $(1.6 \pm 1.0) \times 10^{-3}$ & $53 \pm 17$ & 1.0 & $0.72 / 1$ \\
\hline \multirow{2}{*}{$R_{o}$} & $0.19 \pm 0.08$ & $0.36 \pm 0.07$ & 0.97 & $3.64 / 1$ & $0.028 \pm 0.019$ & $4.1 \pm 2.9$ & 0.84 & $0.11 / 1$ \\
\hline & $0.21 \pm 0.08$ & $0.29 \pm 0.14$ & 1.0 & $3.71 / 1$ & $0.19 \pm 0.05$ & $0.33 \pm 0.10$ & 1.0 & $0.12 / 1$ \\
\hline \multirow{2}{*}{$R_{l}$} & $1.45 \pm 0.03$ & $(9.2 \pm 1.4) \times 10^{-5}$ & 2.72 & $9.4 \times 10^{-9} / 1$ & $1.40 \pm 0.12$ & $(5.7 \pm 2.3) \times 10^{-4}$ & 2.14 & $5.7 \times 10^{-4} / 1$ \\
\hline & $1.16 \pm 0.07$ & $0.030 \pm 0.006$ & 1.0 & $0.35 / 1$ & $1.2 \pm 0.6$ & $0.03 \pm 0.06$ & 1.0 & $4.9 \times 10^{-4} / 1$ \\
\hline
\end{tabular}

$a_{3}$ leads to significant improvement of the fit quality with respect to the quantity for approach (i.2) for $R_{s}$ at statistical errors and especially for $R_{s}$ at all considered types of errors. It should be noted that the difference between smooth curves obtained within approaches (i.1) and (i.2) for BEC radii can be much more noticeable for higher energy FCC than that in Figure 1 which can have a relevant effect on the estimated BEC parameters at FCC.

Dependencies of additional BEC parameters $\mathscr{G}_{2}^{j}\left(\sqrt{s_{\mathrm{NN}}}\right)$, $j=1-4$, for $p+p$ interactions are shown in Figures 2(a)2(d), respectively. Notations of smooth curves correspond to Figure 1; namely, the solid curves show the results calculated with help of fits of BEC radii within approach (i.1) and dashed lines are for special fits (i.2). As seen curves of both types agree with experimental points reasonably for $R_{o} / R_{s}$
(Figure 2(a)) and $R_{m}$ (Figure 2(b)) in total experimentally available energy range. Otherwise approach (i.1) leads to significant overpredictions for $-\delta$ (Figure 2(c)) and $V$ (Figure $2(\mathrm{~d})$ ) at the LHC energy $\sqrt{s_{\mathrm{NN}}}=7 \mathrm{TeV}$ while the curves for special case (i.2) agree with experimental points at this energy.

Thus there is significant uncertainty in functional behavior of dependence of experimental BEC parameters on collision energy due to very limited ensemble of $3 \mathrm{D}$ experimental data for $p+p$ and future experimental results are important for more definitive conclusion with regard to behavior of dependencies $\mathscr{G}_{1,2}^{i}\left(\sqrt{s_{\mathrm{NN}}}\right), i=1-4$.

The pion emission duration $\Delta \tau$ for $p+p$ collisions can be estimated with taking into account the results for $|\delta|$ and kinematic regime for pion pairs under study. $\left\langle\beta_{\perp}\right\rangle \approx 0.82$ 


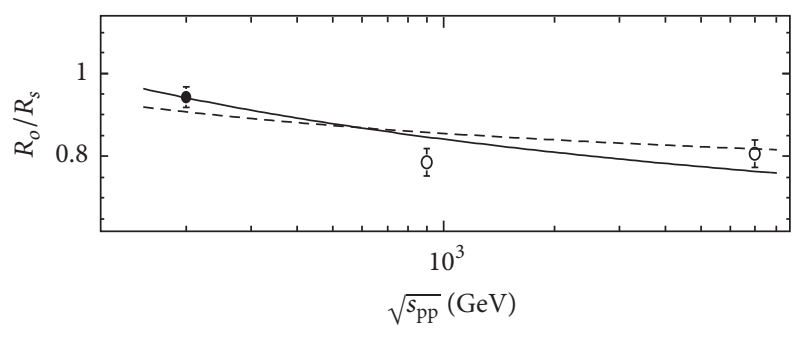

- STAR

- ALICE

(a)

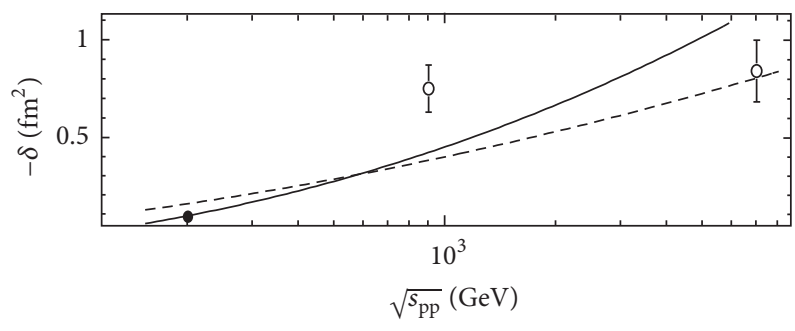

- STAR

- ALICE

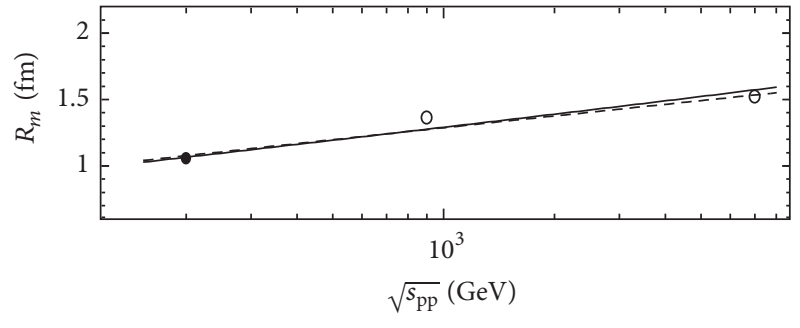

- STAR

- ALICE

(b)

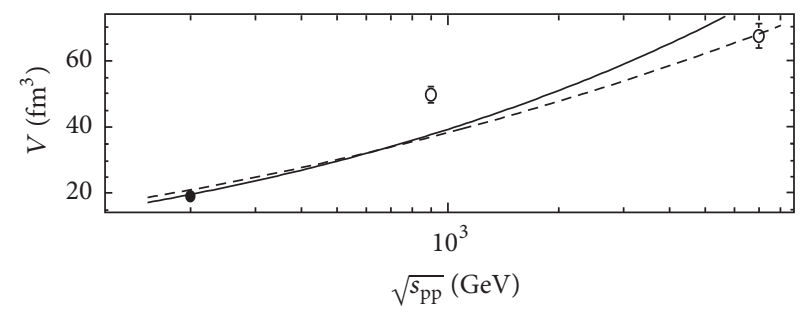

- STAR

- ALICE

(c)

(d)

FIGURE 2: Energy dependence of the additional BEC parameters $\mathscr{G}_{2}^{j}, j=1-4$, for $p+p$ collisions at midrapidity and low $\left\langle k_{T}\right\rangle \simeq 0.2 \mathrm{GeV} / c$. Errors for experimental points are statistical only. Smooth curves are calculated with help of fit results for BEC radii, solid lines are from fits of $R_{i}, i=s, o, l$, by function (7) in case (i.1), and dashed lines correspond to the fits by specific case $R_{i} \propto \ln \varepsilon, i=s, o, l$.

for pion pairs with $\left\langle k_{\perp}\right\rangle \simeq 0.2 \mathrm{GeV} / c$ as well as for nuclear collisions [15]. Then pion emission duration increases from $\Delta \tau=(0.36 \pm 0.08) \mathrm{fm} / \mathrm{c}$ at RHIC energy $\sqrt{s_{\mathrm{pp}}}=0.2 \mathrm{TeV}$ up to $\Delta \tau=(1.25 \pm 0.12) \mathrm{fm} / \mathrm{c}$ at the LHC energy $\sqrt{s_{\mathrm{pp}}}=7 \mathrm{TeV}$ which is the highest for available experimental $\mathrm{BEC}$ results. Thus the pion emission durations in $p+p$ collisions are smaller significantly than that for nuclear interactions [15] in the energy range from RHIC top up to the LHC.

The energy dependence for BEC parameters from the sets $\mathscr{G}_{1,2}^{i}\left(\sqrt{s_{\mathrm{NN}}}\right), i=1-4$, was studied in detail in [15] for nuclear collisions with exception of $R_{m}$ and corresponding scaled quantity $R_{m}^{n}$ defined in the present paper. Energy dependence of these additional BEC parameters is obtained with experimental database for nuclear collisions from [15]. Figures 3 and 4 show the experimental $R_{m}\left(\sqrt{s_{\mathrm{NN}}}\right)$ and $R_{m}^{n}\left(\sqrt{s_{\mathrm{NN}}}\right)$, respectively, as well as smooth curves calculated with fit results for BEC radii at $\sqrt{s_{\mathrm{NN}}} \geq 5 \mathrm{GeV}$ from [15]. In Figures 3 and 4 solid curves for $R_{m}\left(\sqrt{s_{\mathrm{NN}}}\right)$ and for $R_{m}^{n}\left(\sqrt{s_{\mathrm{NN}}}\right)$, respectively, are obtained with help of results from fits of BEC radii by general view of (7) and dashed curves correspond to the calculations with fit results for BEC radii for special case of (7) at $a_{3}=1.0$. As seen in Figures 3 and 4 the behaviors of smooth curves with respect to each other as well as to the experimental data are quite similar for $R_{m}$ and $R_{m}^{n}$. In both cases curves correspond to experimental points reasonably at intermediate energies $10 \leqq \sqrt{s_{\mathrm{NN}}} \lesssim 200 \mathrm{GeV}$ with excess over experimental points in dip region $5 \leqslant \sqrt{s_{\mathrm{NN}}}<10 \mathrm{GeV}$, and opposite situation is seen for $\mathrm{TeV}$ energies (Figures 3 and 4). In Figures 3 and 4 solid curves are close to dashed ones in total energy range considered especially for $R_{m}$ but onset of the excess of solid curve over dashed one at $\sqrt{s_{\mathrm{NN}}}>1 \mathrm{TeV}$ for $R_{m}$ (Figure 3) and at $\sqrt{s_{\mathrm{NN}}}>0.2 \mathrm{TeV}$ for $R_{m}^{n}$ (Figure 4) can lead to a noticeable discrepancy at FCC energy.

As expected, the quantitative comparisons of Figures $1(\mathrm{~b})-1(\mathrm{~d})$ with Figure 2(b) for $p+p$ reactions and Figure 3 with results for BEC radii $R_{i}, i=s, o, l$, in nuclear collisions [15] show that $\forall i=s, o, l: R_{m} \sim R_{i}$ at qualitative level.

Predictions for values of the BEC observables from sets $\mathscr{G}_{1,2}$ are obtained for suggested types of collisions and energies of the LHC and FCC project [4-7] based on the fit results for the main BEC parameters discussed above and in [15]. Estimations are shown in Table 2 for fits by function (7) and its specific case at $a_{3}=1.0$ with inclusion of statistical errors of experimental points, the first column for each type of collisions corresponds to the nominal LHC energy, and the second column corresponds to the energy of FCC project. One notes the fit by constant predicts $\lambda=0.422 \pm 0.004$ for the strength of correlations in $p+p$ collisions for both the LHC and FCC energies. Values for all additional BEC parameters $\mathscr{G}_{2}^{j}, j=1-4$, are calculated with help of its definitions (4)-(6a) and estimations for BEC radii at some energy. The pion emission duration is derived from $|\delta|$ and kinematic regime for pion pairs under study as well as for $p+p$ at lower energies. Results for asymmetric collisions $p+\mathrm{Pb}$ are obtained with help of fit results for scaled BEC parameters [15] and rough estimation $\left\langle R_{\mathrm{pPb}}\right\rangle=(4 \pm 3)$ $\mathrm{fm}$. In the case of $\mathrm{Pb}+\mathrm{Pb}$ collisions the results for main BEC parameters $\mathscr{G}_{1}^{i}, i=1-4$, as well as for $\mathscr{G}_{2}^{j}, j=1,4$, 
TABLE 2: Estimations for space-time characteristic of pion source at the LHC and FCC energies.

\begin{tabular}{|c|c|c|c|c|c|c|}
\hline \multirow{2}{*}{ Parameter } & \multicolumn{2}{|c|}{$p+p, \sqrt{s_{\mathrm{pp}}}(\mathrm{TeV})$} & \multicolumn{2}{|c|}{$p+\mathrm{Pb}, \sqrt{s_{\mathrm{NN}}}(\mathrm{TeV})$} & \multicolumn{2}{|c|}{$\mathrm{Pb}+\mathrm{Pb}, \sqrt{s_{\mathrm{NN}}}(\mathrm{TeV})$} \\
\hline & 14 & 100 & 5.02 & 63 & 5.52 & 39 \\
\hline \multicolumn{7}{|c|}{ From fits by approach (i.1) } \\
\hline$\lambda$ & - & - & $0.16 \pm 0.19$ & $0.05 \pm 0.22$ & $0.41 \pm 0.03$ & $0.40 \pm 0.03$ \\
\hline$R_{s}, \mathrm{fm}$ & $1.8 \pm 0.3$ & $2.3 \pm 0.4$ & $3.9 \pm 2.9$ & $5 \pm 4$ & $6.8 \pm 1.9$ & $8 \pm 3$ \\
\hline$R_{o}, \mathrm{fm}$ & $1.4 \pm 0.6$ & $1.6 \pm 0.7$ & $3.5 \pm 2.6$ & $3.6 \pm 2.7$ & $6.3 \pm 1.0$ & $6.4 \pm 1.0$ \\
\hline$R_{l}, \mathrm{fm}$ & $1.85 \pm 0.07$ & $2.12 \pm 0.11$ & $4 \pm 3$ & $5 \pm 3$ & $7.6 \pm 1.5$ & $8.1 \pm 1.6$ \\
\hline$R_{o} / R_{s}$ & $0.7 \pm 0.4$ & $0.9 \pm 1.0$ & $0.7 \pm 0.7$ & $0.7 \pm 0.7$ & $0.9 \pm 0.3$ & $0.8 \pm 0.3$ \\
\hline$R_{m}, \mathrm{fm}$ & $1.67 \pm 0.27$ & $2.0 \pm 0.3$ & $3.9 \pm 1.7$ & $4.4 \pm 1.9$ & $6.9 \pm 0.9$ & $7.6 \pm 1.2$ \\
\hline$\delta, \mathrm{fm}^{2}$ & $-1.5 \pm 2.0$ & $-2.7 \pm 2.9$ & $-3 \pm 29$ & $-20 \pm 50$ & $-6 \pm 29$ & $-30 \pm 60$ \\
\hline$\Delta \tau, \mathrm{fm} / \mathrm{c}$ & $1.5 \pm 1.0$ & $2.0 \pm 1.1$ & $2 \pm 11$ & $5 \pm 7$ & $3 \pm 7$ & $7 \pm 7$ \\
\hline$V, \mathrm{fm}^{3}$ & $(1.0 \pm 0.3) \times 10^{2}$ & $(1.8 \pm 0.5) \times 10^{2}$ & $(1.0 \pm 1.7) \times 10^{3}$ & $(2 \pm 3) \times 10^{3}$ & $(6 \pm 3) \times 10^{3}$ & $(9 \pm 8) \times 10^{3}$ \\
\hline \multicolumn{7}{|c|}{ From fits by approach (i.2) } \\
\hline$\lambda$ & $0.42 \pm 0.11$ & $0.42 \pm 0.13$ & $0.097 \pm 0.004$ & - & $0.362 \pm 0.009$ & $0.315 \pm 0.011$ \\
\hline$R_{s}, \mathrm{fm}$ & $1.7 \pm 0.7$ & $2.0 \pm 0.8$ & $3.3 \pm 2.4$ & $3.5 \pm 2.6$ & $5.79 \pm 0.10$ & $6.11 \pm 0.12$ \\
\hline$R_{o}, \mathrm{fm}$ & $1.4 \pm 0.7$ & $1.6 \pm 0.9$ & $3.6 \pm 2.6$ & $3.7 \pm 2.7$ & $6.49 \pm 0.12$ & $6.74 \pm 0.15$ \\
\hline$R_{l}, \mathrm{fm}$ & $1.82 \pm 0.17$ & $1.96 \pm 0.20$ & $5 \pm 3$ & $5 \pm 4$ & $8.20 \pm 0.16$ & $9.04 \pm 0.19$ \\
\hline$R_{o} / R_{s}$ & $0.8 \pm 0.5$ & $0.8 \pm 0.5$ & $1.1 \pm 1.1$ & $1.1 \pm 1.1$ & $1.12 \pm 0.03$ & $1.10 \pm 0.03$ \\
\hline$R_{m}, \mathrm{fm}$ & $1.6 \pm 0.4$ & $1.9 \pm 0.4$ & $3.7 \pm 1.6$ & $4.0 \pm 1.7$ & $6.75 \pm 0.07$ & $7.19 \pm 0.09$ \\
\hline$\delta, \mathrm{fm}^{2}$ & $-1 \pm 3$ & $-2 \pm 4$ & $2 \pm 25$ & $1 \pm 27$ & $8.7 \pm 2.0$ & $8.2 \pm 2.4$ \\
\hline$\Delta \tau, \mathrm{fm} / \mathrm{c}$ & $1.2 \pm 1.9$ & $1.5 \pm 2.1$ & $2 \pm 11$ & $1 \pm 14$ & $3.6 \pm 0.4$ & $3.5 \pm 0.5$ \\
\hline$V, \mathrm{fm}^{3}$ & $80 \pm 60$ & $(1.3 \pm 1.0) \times 10^{2}$ & $(0.8 \pm 1.3) \times 10^{3}$ & $(1.0 \pm 1.6) \times 10^{3}$ & $(4.32 \pm 0.17) \times 10^{3}$ & $(5.31 \pm 0.23) \times 10^{3}$ \\
\hline
\end{tabular}

and emission duration are from [15]; furthermore the brief discussion of estimations for these BEC parameters at the LHC and FCC energies can be also found in the previous studies $[15,29]$. As seen from Table 2 all BEC parameters have values coincided with each other for two approaches (i.1) and (i.2) within errors for corresponding collision energies and types of strong interaction processes. In general estimations for BEC parameters calculated with approach (i.1) do not change from the LHC up to FCC energies within large uncertainties for all collisions under consideration. Protonproton and nucleus-nucleus collisions are characterized by similar strength of correlations for approach (i.2) for the LHC and FCC energies, and the large uncertainty for $p+$ $\mathrm{Pb}$ allows only the qualitative conclusion that $\lambda$ is somewhat smaller for this type of collision than that for $\mathrm{Pb}+\mathrm{Pb}$ in the case of general view of (7) and, for $p+p, \mathrm{~Pb}+\mathrm{Pb}$ within the framework of approach (i.2). $\lambda$ is quite constant for $p+p$ collisions but shows noticeable decrease for heavy ion collisions at increasing of $\sqrt{s_{\mathrm{NN}}}$ in the energy range LHC-FCC for fit by specific case of (7) at $a_{3}=1.0$. For $p+\mathrm{Pb}$ the estimation of $\lambda$ obtained with approach (i.1) at $\sqrt{s_{\mathrm{NN}}}=5.02 \mathrm{TeV}$ agrees rather well with experimental result [31] while approach (i.2) underpredicts the strength of correlations at the LHC energy. Furthermore estimations for $\mathrm{BEC}$ radii of pion source produced in $p+\mathrm{Pb}$ collisions at $\sqrt{s_{\mathrm{NN}}}=5.02 \mathrm{TeV}$ (Table 2 ) agree with experimental results $[31,32]$ within large errors. The space scales are about $2 \mathrm{fm}$ in $p+p$, about 4-5 fm in $p+\mathrm{Pb}$, and 6-9 fm in $\mathrm{Pb}+\mathrm{Pb}$ collisions for pion source at FCC energies. In the case of estimations for BEC radii obtained with general view of (7) large uncertainties do not allow the definite conclusion and one can see qualitative indication only that $R_{o}$ is somewhat smaller than other radii in all collision types for both the LHC and FCC energies. For estimations based on the special case of (7) with $a_{3}=1.0$ there is noticeable increase of all BEC radii for transition from the LHC to FCC energy in heavy ion collision (Table 2). As a consequence $R_{m}$ and $V$ are larger at $\sqrt{s_{\mathrm{NN}}}=39 \mathrm{TeV}$ than that at the LHC energy $\sqrt{s_{\mathrm{NN}}}=5.52 \mathrm{TeV}$. Spread of values of BEC radii leads to significant uncertainties for estimations of additional spacetime parameters especially for extremely asymmetric $p+\mathrm{Pb}$ collisions for which the large error for $\left\langle R_{\mathrm{pPb}}\right\rangle$ increases greatly uncertainties for BEC quantities in Table 2. Consequently the volume of the pion source can be roughly estimated as about $200 \mathrm{fm}^{3}$ in $p+p, 2000 \mathrm{fm}^{3}$ in $p+\mathrm{Pb}$, and $10^{4} \mathrm{fm}^{3}$ in $\mathrm{Pb}+\mathrm{Pb}$ collisions at FCC energies in comparison with $100 \mathrm{fm}^{3}$ in $p+p, 1000 \mathrm{fm}^{3}$ in $p+\mathrm{Pb}$, and $6000 \mathrm{fm}^{3}$ in $\mathrm{Pb}+\mathrm{Pb}$ at the nominal LHC energies. These estimations indicate the consistent growth of $V$ for transition from the small system collisions to the $\mathrm{Pb}+\mathrm{Pb}$. For approach (i.1) estimations for all parameters $\mathscr{G}_{2}^{j}, i=j-4$, and for emission duration do not depend on energy in the range LHC-FCC for all types of collisions within errors. This conclusion is also valid for approach (i.2) with exception of $R_{m}$ and $V$ for $\mathrm{Pb}+\mathrm{Pb}$ discussed above. It should be noted that weak change of main BEC parameters $\mathscr{G}_{1}^{i}, i=1-4$, is qualitatively expected for energy domain from the LHC up to FCC because of general trends of available experimental points and consequent slow logarithmic increase with collision energy for smooth analytic functions used in the present analysis as well as in $[15,29]$. 


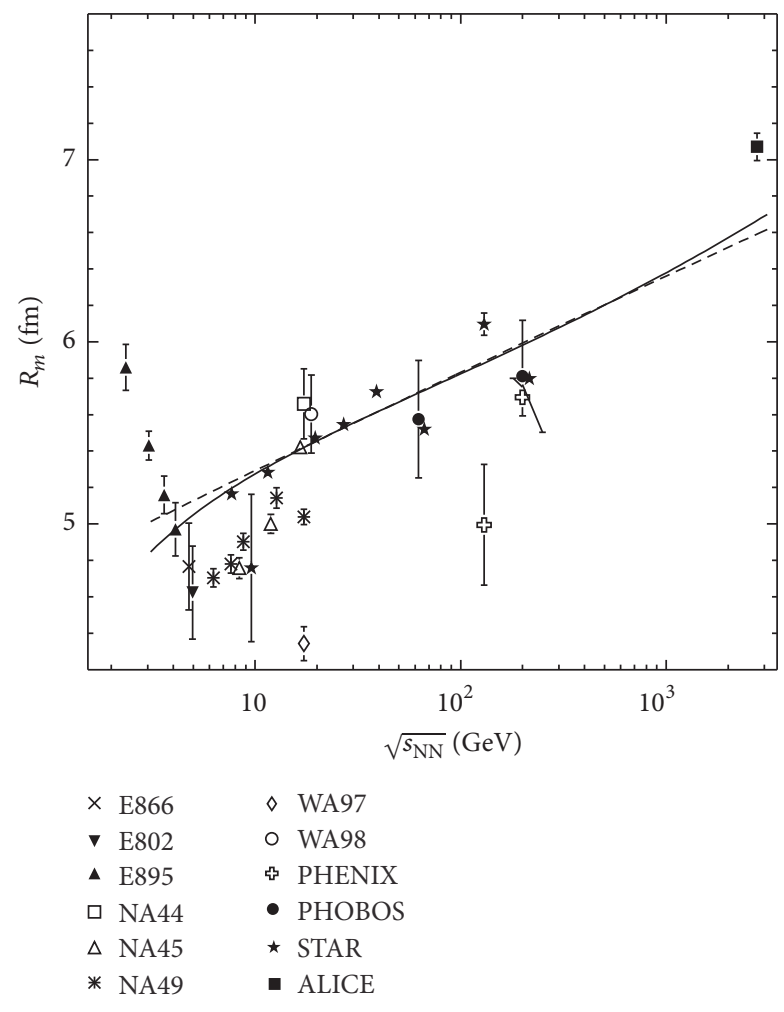

FIgURE 3: Energy dependence of $R_{m}$ for secondary charged pions in central heavy ion collisions $\mathrm{Au}+\mathrm{Au}, \mathrm{Au}+\mathrm{Pb}$, and $\mathrm{Pb}+\mathrm{Pb}$ in midrapidity region and at $\left\langle k_{\perp}\right\rangle \simeq 0.2 \mathrm{GeV} / c$. Error bars are only statistical (for NA44, total uncertainties). Smooth curves are derived from (4) and the fit results for BEC radii [15] without the point of the WA97 experiment [16]. The solid line corresponds to the fits of $\mathrm{BEC}$ radii by function (7) and dashed line to the fits by specific case $\forall i=s, o, l: R_{i} \propto \ln \varepsilon$.

\section{Pion Laser at FCC Energies}

Results shown above for space-time extent of pion emissions region allow the study of possibility of Bose-Einstein condensation with consequent formation of pion laser in high energy strong interaction processes. The key quantity is the charged particle density which is defined as follows:

$$
n_{\mathrm{ch}}=\frac{N_{\mathrm{ch}}}{V},
$$

where $N_{\mathrm{ch}}$ is the total charged particle multiplicity and $V$ is the source volume at freeze-out stage. The critical density $n_{\mathrm{ch}}^{c}$ can be calculated with help of (8) and transition to the critical total multiplicity $N_{\mathrm{ch}} \rightarrow N_{\mathrm{ch}}^{c}$. The last multiplicity parameter was derived for $1 \mathrm{D}$ thermal Gaussian distribution in [13]. Within the 3D Gaussian parametrization for source the following relation is suggested:

$$
N_{\mathrm{ch}}^{c}=\eta^{-1}\left[\left(\Delta R_{m}\right)^{2}+\sqrt{\left(p_{0} R_{m}\right)^{2}+0.25}\right]^{3 / 2} \text {. }
$$

Here $\eta=0.25$ is the fraction of the pions to be emitted from a static Gaussian source, $\Delta=0.25 \mathrm{GeV} / \mathrm{c}$ is a momentum spread and $p_{0} / T=p^{2} / 2 \Delta^{2}[13], T$ is source temperature

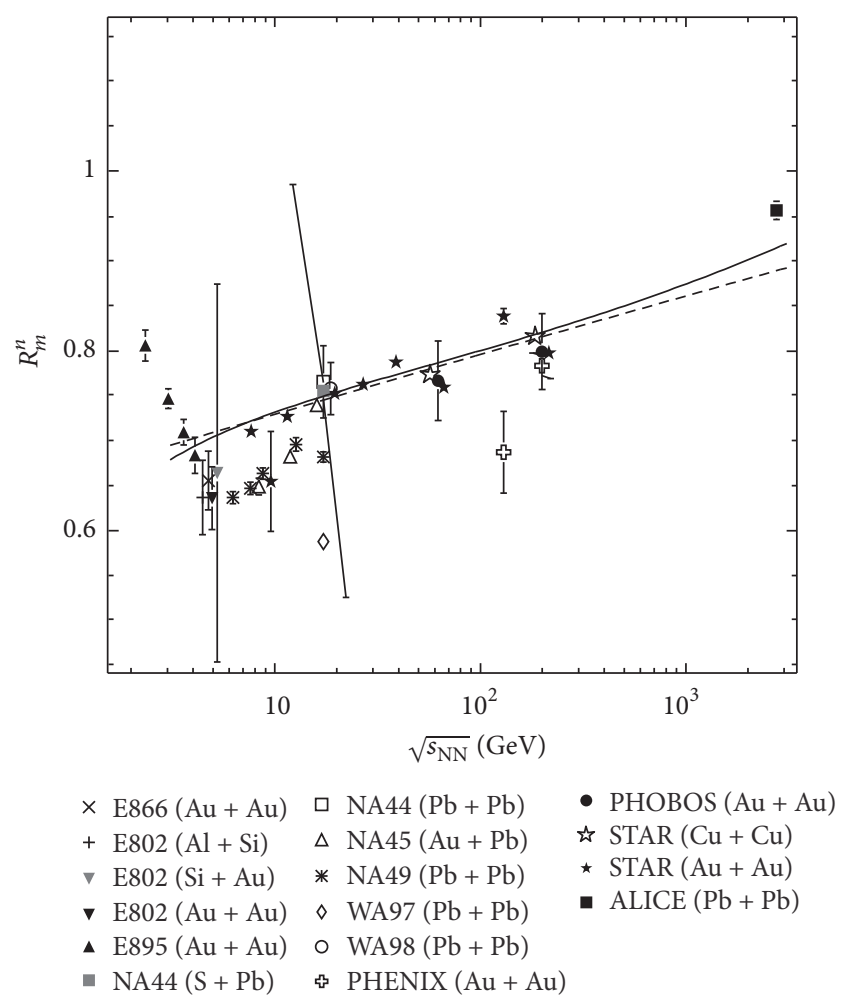

FIGURE 4: Energy dependence of scaled $R_{m}^{n}$ for secondary charged pions in various nucleus-nucleus collisions at $\left\langle k_{\perp}\right\rangle \simeq 0.2 \mathrm{GeV} / c$. Experimental results are shown for central collisions (for minimumbias event in the case of $\mathrm{E} 802$ for $\mathrm{Al}+\mathrm{Si}$ ), for pairs of $\pi^{-}$mesons (in the cases of ALICE and STAR for both the $\mathrm{Cu}+\mathrm{Cu}$ and $\mathrm{Au}+$ Au at $\sqrt{s_{\mathrm{NN}}}=7.7-62.4$ and $200 \mathrm{GeV}$, for $\pi^{ \pm} \pi^{ \pm}$pairs, E802 for $\mathrm{Al}+$ $\mathrm{Si}$ and NA44 for $S+\mathrm{Pb}$, for pairs of $\pi^{+}$mesons) and for standard Coulomb correction $P_{C}^{(1)}(q)$ (in the cases of ALICE, NA44, NA45, $\mathrm{PHOBOS}$, and STAR for both the $\mathrm{Cu}+\mathrm{Cu}$ and $\mathrm{Au}+\mathrm{Au}$ at $\sqrt{s_{\mathrm{NN}}}=$ 7.7, 11.5-62.4, and $200 \mathrm{GeV}$, for correction $\left.P_{C}^{(3)}\right)$. Statistical errors are shown (for NA44, total uncertainties). Smooth curves are derived from fit results for scaled BEC radii [15]. The solid line corresponds to the fits of scaled BEC radii by function (7) and dashed line to the fits by specific case $\forall i=s, o, l: R_{i}^{n} \propto \ln \varepsilon$.

supposed to be equal to the parameter value at chemical freeze-out, and it is suggested $p \approx 3 T$ [33]. It should be emphasized that there are no qualitative studies of $T\left(\sqrt{s_{\mathrm{pp}}}\right)$ for $p+p$ collisions so far but set of results for mean multiplicity and the pseudorapidity density at midrapidity for charged particles in various collisions [17, 34-36] as well as recent results for deconfinement in small system [37, 38] indicate remarkable similarity of both the bulk and the thermodynamic properties of strongly interacting matter created in high energy $p+p / \bar{p}+p$ and $\mathrm{A}+\mathrm{A}$ collisions. Therefore the hypothesis is suggested for similar energy dependence of $T$ in both $p+p$ and $A+A$ interactions with taking into account $\sqrt{s_{\mathrm{pp}}} \simeq 3 \sqrt{s_{\mathrm{NN}}}[17,34-36]$ and consequently the analytic energy dependence of $T$ from $[39,40]$ is used for all types of strong interaction processes considered in this section. Thus appropriate analytic function is derived for energy dependence of $N_{\mathrm{ch}}^{c}$. Also $N_{\mathrm{ch}}$ versus collision energy 
in (8) is defined by some smooth approximations which are specified below for $p+p$ and $\mathrm{A}+\mathrm{A}$ collisions. Experimental estimations for $V$ available in Figure 2(e) for $p+p$ and in [15] for $\mathrm{A}+\mathrm{A}$ collisions can be used for calculations of $n_{\mathrm{ch}}$ at certain energies. The results of such calculations are called experimental points and marked by symbols in Figures 5 and 6 in the sense that BEC measurements are used in these cases. As seen from Table 1 for $p+p$ and from [15] for heavy ion collisions the smooth energy dependence of source volume can be calculated with help of the fits of BEC radii by (ii.1) general function (7) as well as (ii.2) by specific case $R_{i} \propto \ln \varepsilon, i=s, l$. Relation (6a) for source volume is used in (8) for experimental estimations as well as for calculations of smooth energy dependence of $n_{\mathrm{ch}}$ in both $p+p$ and $\mathrm{A}+\mathrm{A}$ collisions. On the other hand it seems reasonable to use (6b) for estimation of critical charged density because relation (9) with $R_{m}$ is only available. Taking into account the qualitative relation between $R_{m}$ and $3 \mathrm{D}$ BEC radii shown above one expects that the difference of numerical factors for two definitions of $V$ (6a) and ( $6 \mathrm{~b}$ ) provides additional uncertainty $\sim 3 \sqrt{\pi / 2}$ in the ratio $n_{\mathrm{ch}} / n_{\mathrm{ch}}^{c}$. Therefore theoretical investigations are essential for quantitative account for geometry of source and decrease of uncertainty due to calculation of volume of emission region. As it follows from (3) and discussion in Section 2 relation (8) defines the upper boundary for true value of charged particle density

$$
n_{\mathrm{ch}}=\sup n_{\mathrm{ch}}^{\mathrm{tr}}
$$

Strictly speaking and possibly the discrepancy between estimation from (8) and $n_{\mathrm{ch}}^{\text {tr }}$ will be increased with growth of collision energy due to intensification of longitudinal and radial collective flows. During the recent years the collectivity in small colliding systems is under intensive theoretical [4148] and experimental studies [49-51] but the results obtained already mean that the statement above is valid for $p+p$ interactions as well as for nucleus-nucleus collisions in TeVenergy domain at least. In general one can assume the weaker dependence of $n_{\mathrm{ch}}^{c}$ on space-time parameters of particle source compared to that for $n_{\text {ch }}$ due to BEC radius in (9). The uncertainties of $N_{\mathrm{ch}}$ calculated by standard way from errors of fit parameters are attributed as statistical errors. The statistical uncertainties for $N_{\mathrm{ch}}^{c}$ are estimated by standard way at assigned relative error $\delta \Delta=0.05$ and with taking into account the errors for fits of $R_{m}$ and $T$ used while systematic uncertainties are deduced by varying of $\eta$ within the range $\eta=0.20-0.30$ only. The statistical errors of $V$ propagated from corresponding uncertainties of BEC radii are only taken into account below.

Total charged multiplicity $N_{\mathrm{ch}}^{\mathrm{pp}}$ is calculated within various approaches [17-19]. In Figure 5 energy dependence is shown for $n_{\mathrm{ch}}^{\mathrm{pp}}$ as well as for critical particle density. It should be noted that in the case of $p+p$ collisions minimumbias events are used in the BEC analyses $[52,53]$ and these events correspond to the non-singly diffractive (NSD) $p+p$ collisions at $\sqrt{s_{\mathrm{pp}}}=0.2 \mathrm{TeV}$ [37] as well as at the LHC energies $[54,55]$. Thus experimental points are obtained for $N_{\mathrm{ch}}^{\mathrm{pp}}$ calculated with hybrid approximation [17]. The thick

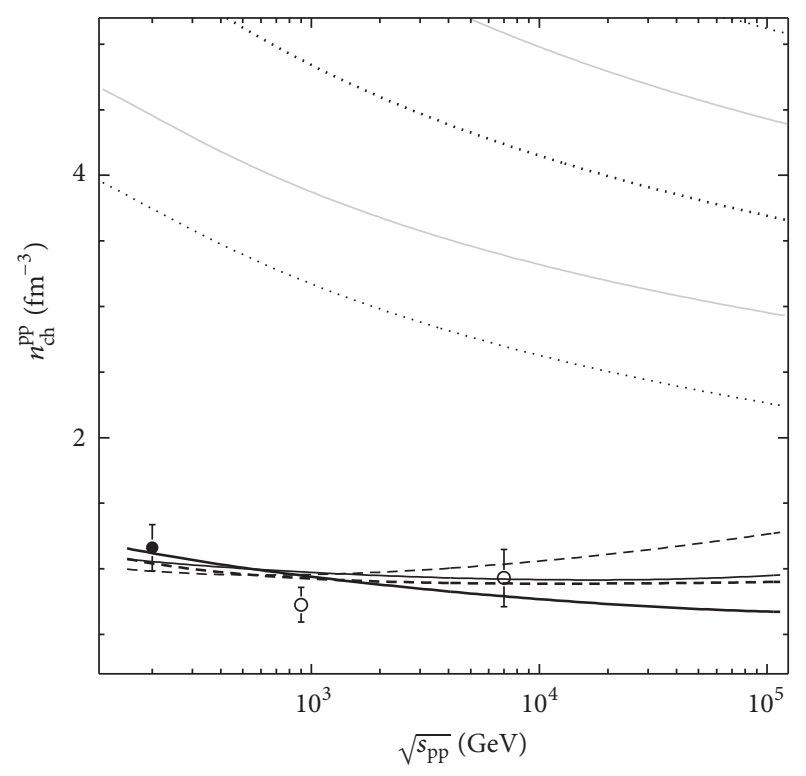

The freeze-out volume from BEC data:

- STAR

- ALICE

FIGURE 5: Energy dependence of estimations for charged particle density and for critical one in $p+p$ collisions. Points are calculated with help of the hybrid function for $N_{\mathrm{ch}}$ [17] and the experimental estimations for $V$ and uncertainties for points are propagated from statistical errors of measurements and fits used. Solid lines correspond to the hybrid approximation of $N_{\mathrm{ch}}$ [17] and dashed lines are for 3NLO pQCD equation [18] while thick lines show results with $V$ calculated with the fits of BEC radii by (7) in case (ii.1) and thin lines with the fits by specific case $R_{i} \propto \ln \varepsilon, i=s, l$. Critical charged particle density is shown by dotted line with its statistical uncertainty levels represented by thin dotted lines. The heavy grey lines correspond to the systematic \pm 1 s.d. of $n_{\mathrm{ch}}$ calculated by varying of $\eta$ on \pm 0.05 .

solid and dashed lines correspond to the source volume within approach (ii.1) and thin lines correspond to $V$ from case (ii.2). In Figure 5 results for $n_{\mathrm{ch}}^{\mathrm{pp}}$ are shown by solid lines for hybrid approximation of total charged multiplicity [17] and dashed lines are deduced with $N_{\mathrm{ch}}^{\mathrm{pp}}$ within $3 \mathrm{NLO}$ pQCD approach [18] at $\sqrt{s_{\mathrm{pp}}} \simeq 0.35 \sqrt{\boldsymbol{s}_{\mathrm{ee}}}[34-36,56]$ and parameters from [57] for number of colors $N_{c}=3$. Available experimental estimations show almost constant $n_{\mathrm{ch}}^{\mathrm{pp}} \simeq 1$. Smooth curves agree with experimental points reasonably for any approximation of $N_{\mathrm{ch}}^{\mathrm{pp}}$ and $V$. As seen the differences between various approaches for each of the two parameters are small up to the LHC energy and increase for FCC noticeably. The dependence of critical particle density $n_{\mathrm{ch}}^{c, \mathrm{pp}}\left(\sqrt{s_{\mathrm{pp}}}\right)$ shown by dotted line decreases with energy. The statistical \pm 1 s.d. band limits are drawn by thin dotted line while this large uncertainty is mostly dominated by the precision of BEC parameters of emission region. The systematic \pm 1 s.d. boundaries are shown by heavy grey lines. As seen from Figure $5 n_{\text {ch }}$ is smaller than its critical value in $p+p$ collisions up to FCC energy $\sqrt{s_{\mathrm{pp}}}=100 \mathrm{TeV}$ for any approaches for total charged multiplicities and $V$ under study. 
This conclusion is valid even with taking into account large statistical uncertainties. Thus one can not expect the kind of lasing behavior for secondary pions in $p+p$ collisions within the present approach.

One can note the coincidence between the experimental values of $N_{\mathrm{ch}}$ in $p+p$ and $\bar{p}+p$ collisions at energies 0.2 and $0.9 \mathrm{TeV}$ and general smooth energy dependence of $N_{\mathrm{ch}}$ for NSD events in the interactions under discussion [55]. Also reasonable agreement is observed for pseudorapidity density $d N_{\text {ch }} / d \eta$ measured in $p+p[54,55,58]$ and $\bar{p}+p[59,60]$ interactions at energies indicated above. But there are no 1D BEC analyses with Gaussian model in $\bar{p}+p$ collisions at $\sqrt{s_{\overline{\mathrm{pp}}}}=0.2$ and $0.9 \mathrm{TeV}$. Furthermore the quantitative comparison of BEC results from $p+p$ to those from $\bar{p}+p$ collisions is difficult for $1 \mathrm{D}$ case due to limited ensemble of experimental results in the last case [20] and noticeable difference between collision energies in $p+p$ and $\bar{p}+p$ for available BEC measurements; there is no BEC analysis with 3D Gaussian model for $\bar{p}+p$ so far. On qualitative level close values for multiplicity quantities can be expected in $p+p$ and $\bar{p}+p$ collisions in particular at energies about $2 \mathrm{TeV}$ while the

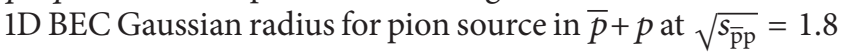
and $1.96 \mathrm{TeV}[61,62]$ is significantly larger than that from $p+p$ at $\sqrt{s_{\mathrm{pp}}}=2.36 \mathrm{TeV}$ [63]. Therefore $n_{\mathrm{ch}}$ is expected to be smaller in $\bar{p}+p$ than that in $p+p$ collisions at least in $\mathrm{TeV}$ energy domain at close values of critical quantity due to its weaker dependence on space-time extent of particle source. Thus the pion lasers seem impossible in high energy $\bar{p}+p$ collisions within the rough assumptions.

As discussed above the estimations of space-time extent of pion source are characterized by large uncertainties; moreover development of equation for critical parameters for multidimensional (3D) case seems important for improvement of precision of studies and for more certain conclusions. The future quantitative experimental and theoretical investigations are essential for verification of the results shown above and possibility of novel coherent effects in different types of collisions in high energy domain.

Total charged multiplicity $N_{\mathrm{ch}}^{\mathrm{AA}}$ is calculated with hybrid equations $[17,19]$. Figure 6 demonstrates energy dependence for both $n_{\mathrm{ch}}^{\mathrm{AA}}$ and the critical particle density where smooth approximation for $n_{\mathrm{ch}}^{\mathrm{AA}}$ is shown by solid line for hybrid approximation of total charged multiplicity [17] and by dashed line for $N_{\mathrm{ch}}^{\mathrm{AA}}$ from [19], and experimental points are obtained for $N_{\mathrm{ch}}^{\mathrm{AA}}$ calculated with equation from [19]. Both the curves for $n_{\mathrm{ch}}^{\mathrm{AA}}$ and experimental points are deduced with mean number of participants $\left\langle N_{\text {part }}\right\rangle=382.8 \pm 3.1$ which corresponds to the $0-5 \%$ central $\mathrm{Pb}+\mathrm{Pb}$ collisions [19]. This simplest approach seems reasonable because heavy ion collisions are only considered in Figure 6. The source volume is calculated within approach (ii.1) and results for $\mathrm{BEC}$ radii in nuclear collisions [15]. Experimental points for $n_{\mathrm{ch}}^{\mathrm{AA}}$ increase with $\sqrt{s_{\mathrm{NN}}}$ and agree reasonably with smooth curves for both parameterizations of total charged multiplicity under consideration. Comparison between particle densities in $p+p$ (Figure 5) and A + A (Figure 6) strong interaction processes indicates the enhancement of $n_{\mathrm{ch}}^{\mathrm{AA}}$ over

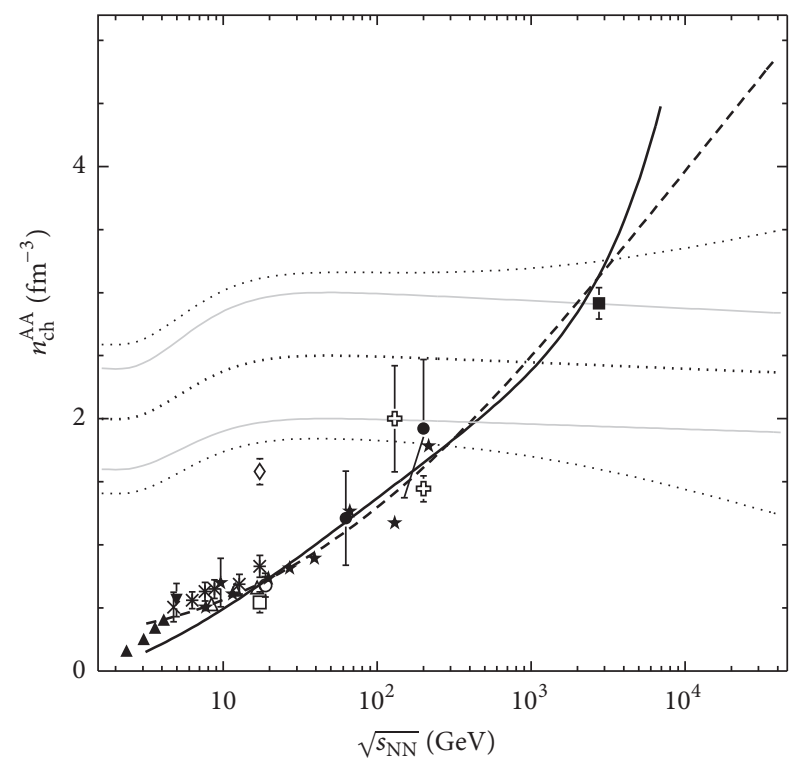

The freeze-out volume from BEC data:

$\times$ E866 $\diamond$ WA97

V E802 O WA98

$\triangle$ E895 \& PHENIX

$\square$ NA44 $\bullet$ PHOBOS

$\triangle$ NA45 $\quad$ STAR

* NA49 - ALICE

FIGURE 6: Energy dependence of estimations for charged particle density and for critical one in A + A collisions. Points are calculated with help of the hybrid function for $N_{\mathrm{ch}}$ [19] and the experimental estimations for $V$ and uncertainties for points are propagated from statistical errors of measurements and fits used. Solid line corresponds to the hybrid approximation of $N_{\mathrm{ch}}$ [17] and dashed line is for parametrization of total charged multiplicity from [19]. Critical charged particle density is shown by dotted line with its statistical uncertainty levels represented by thin dotted lines. The heavy grey lines correspond to the systematic \pm 1 s.d. of $n_{\mathrm{ch}}$ calculated by varying of $\eta$ on \pm 0.05 .

$n_{\mathrm{ch}}^{\mathrm{pp}}$ starting with RHIC energy $200 \mathrm{GeV}$ per nucleon-nucleon pair; furthermore this enhancement increases with growth of collision energy. The critical particle density $n_{\mathrm{ch}}^{c, \mathrm{AA}}$ shown by dotted line depends weakly on $\sqrt{s_{\mathrm{NN}}}$ in nuclear collisions. In Figure 6 the line types for statistical and systematic \pm 1 s.d. band limits are the same as well as for corresponding smooth curves for $p+p$ collisions (Figure 5). The statistical uncertainty driven by the precision of BEC parameters of emission region increases noticeably for multi-TeV region $\sqrt{s_{\mathrm{NN}}} \gtrsim 10 \mathrm{TeV}$ in $\mathrm{A}+\mathrm{A}$ interactions. The situation changes dramatically with transition from $p+p$ to nuclear collisions at high energies. As seen from Figure 6 the following relation $n_{\mathrm{ch}}^{\mathrm{AA}} \approx n_{\mathrm{ch}}^{c, \mathrm{AA}}$ is valid at RHIC and the LHC energies within wide uncertainty band for critical value of charged particle density. Furthermore there is clear enhancement of smooth curves for $n_{\mathrm{ch}}^{\mathrm{AA}}$ over $n_{\mathrm{ch}}^{c, \mathrm{AA}}$ at $\sqrt{s_{\mathrm{NN}}} \gtrsim 10 \mathrm{TeV}$ with taking into account large statistical uncertainty for $n_{\mathrm{ch}}^{c, \mathrm{AA}}\left(\sqrt{s_{\mathrm{NN}}}\right)$. Thus one can expect the appearance of novel effects dominated by Bose-Einstein condensation in nucleus-nucleus collisions at 
FCC energy. In particular, Figure 6 indicates the possibility for pion laser effect in heavy ion collisions at $\sqrt{s_{\mathrm{NN}}} \gtrsim 10 \mathrm{TeV}$ within the approach under study.

With theory point of view the conception of the pion laser was intensively studied within the framework of the model of independent factorized sources [13, 14, 64-66] as well as in the model of disoriented chiral condensate (DCC) decay [67]. On the other hand possible experimental signatures of Bose-Einstein condensation, in particular, the pion laser effect in heavy ion collisions at FCC energy, should be the subject of future detailed investigations. Here one notes the following experimental signatures of Bose-Einstein condensation. In general, one can expect enhancement of high-multiplicity events $[13,14]$ and the decrease of chaoticity parameter derived from two-particle BEC analysis due to amplification of coherent particle production [68]. The effects of multiboson symmetrization regarding isospin fluctuations can manifest itself through enhancement of the events with anomalous isospin imbalance like CENTAURO events in high energy cosmic ray $[13,14]$. The shrinkage of the BEC radius is the more specific prediction within the model of the DCC decay when the Bose-Einstein condensation takes place [67]. This effect potentially represents one of the most pronounced features of the pion laser, because the available experimental BEC radii show smooth increase with collision energy both in $p+p$ interactions (Figure 1) and the heavy ion collisions $[15,29]$.

\section{Study of Correlation Peak Shape}

The accelerator parameters within the FCC project [4-7] open the new possibility for detailed study of peak structure for two-particle BEC correlation function. The peak of CF is described by $\mathbf{K}_{2}^{\mathrm{ph}}(\mathbf{A})$. In general there is rich class of random processes with additive stochastic variables for which (i.e., for these processes) there are finite distributions but the Central Limit Theorem (CLT) in the traditional (Gaussian) formulation is not valid. The class of random processes under consideration are characterized by large fluctuations, powerlaw behavior of distributions in the range of large absolute values of random variables, and nonanalytic behavior of characteristic function of the probability distribution for small values of its arguments [69]. In mathematical statistics and probability theory the class of such distributions are called stable (on Lévy) distributions (in literature for physics and mathematics the multidimensional distributions included in the class are called Lévy-Feldheim distributions) [70, 71]. The general stable distribution is described by four parameters: an index of stability (or Lévy index) $\alpha \in(0,2]$, a parameter of skewness $\beta$, scale $\gamma$, and location $\delta$. These distributions satisfy requirements of generalized Central Limit Theorem (gCLT) and self-similarity (the applications of stable distributions in the physics of fundamental interactions and, in particular, for correlation femtoscopy are described, e.g., in [26]). Therefore the detailed investigation of the shape of correlation peak has to do with verification of hypothesis of possible selfaffine fractal-like geometry of emission region. At present the study of Lévy-Feldheim distributions is the advanced region of mathematics but the specific case of central-symmetrical stable distributions is known in more detail [72]. Just this subclass of stable distributions is most important on the point of view of investigation for BEC. In this case the application of subset of nonisotropic central-symmetrical Lévy-Feldheim distributions [73] seems reasonable because the projections of the $3 \mathrm{D}$ relative momentum $\vec{q}$ are independent random variables.

The multidimensional generalized parametrization of $n$th order for CF (1a) and (1b) can be written as follows [11]:

$$
\begin{array}{r}
C_{2}^{\mathrm{ph}, n}(q, K)=\xi_{1}(q, K)\left[1+\xi_{2}(q, K) \mathbf{K}_{2}^{\mathrm{ph}, n}(\mathbf{A})\right], \\
\mathbf{K}_{2}^{\mathrm{ph}, n}(\mathbf{A})=\mathbf{K}_{2}^{\mathrm{ph}, 0}(\mathbf{A}) \prod_{i=1}^{3} \prod_{j=1}^{3}\left[1+\sum_{m=1}^{n} g_{m} h_{m}\left(A_{i j}\right)\right], \\
\text { at } n \geq 1,
\end{array}
$$

where $\mathbf{K}_{2}^{\mathrm{ph}, n}$ is phenomenological parametrization of $n \mathrm{th}$ order for $\mathrm{cCF}(2)$ and functions $\xi_{1,2}(q, K)$ take into account formally all corrections on degree of source chaoticity, final state interactions, and so forth. The experimental and theoretical investigations in the field of $\mathrm{BEC}$ allow us to derive some approach for cumulant two-particle function (2) in the lowest order. Within the framework of the subset of nonisotropic central-symmetrical Lévy-Feldheim distributions the most general parametrization of $\mathbf{K}_{2, L}^{\mathrm{ph}, 0}$ can be given by

$$
\begin{aligned}
\mathbf{K}_{2, L}^{\mathrm{ph}, 0}(\mathbf{A}) & =\prod_{i=1}^{3} \prod_{j=1}^{3} \mathbf{K}_{2, L}^{\mathrm{ph}, 0}\left(A_{i j}\right) \\
& =\exp \left(-\sum_{i, j=1}^{3}\left|A_{i j}\right|^{\alpha / 2}\right), \\
\mathbf{K}_{2, L}^{\mathrm{ph}, 0}(x) & =\exp \left(-|x|^{\alpha / 2}\right) .
\end{aligned}
$$

Here were take into account that $x \equiv\left(q_{i} R_{i}\right)^{2}, i=$ $l, o, s$, for correlation femtoscopy, the products are on the space components of vectors. $\left.\left\{h_{n}(x)\right\}\right|_{n=0} ^{\infty}$ is the closed system of orthogonal polynomials in the Hilbert space $\mathscr{H}$ : $\int d x \mathbf{K}_{2}(x) h_{n}(x) h_{m}(x)=\delta_{n m}, g_{n}=\int d x \mathbf{K}_{2}(x) h_{n}(x)$. The system $\left.\left\{h_{n}(x)\right\}\right|_{n=0} ^{\infty}$ for exponential weight function can be derived with the help of the following recurrent relations $a_{1} h_{1}(x)=\left(x-b_{0}\right) h_{0}(x), a_{n+1} h_{n+1}(x)=\left(x-b_{n}\right) h_{n}(x)-$ $a_{n-1} h_{n-1}(x), n=1,2, \ldots$ [73-77], and moments $\mu_{n}=$ $\int_{-\infty}^{\infty} d x x^{n} \exp \left(-|x|^{\gamma}\right)=2 \gamma^{-1} \Gamma\left(\gamma^{-1}[n+1]\right), n \geq 0, \gamma>0$ [78]. Here $\forall n \geq 0: b_{n}=\widetilde{H}_{n+1} H_{n+1}^{-1}-\widetilde{H}_{n} H_{n}^{-1} ; \forall n>$ $0: a_{n}=H_{n}^{-1} \sqrt{H_{n-1} H_{n+1}}$, and $H_{n}$ and $\widetilde{H}_{n}$ are the following determinants:

$$
H_{n}=\left|\begin{array}{ccc}
\mu_{0} & \cdots & \mu_{n-1} \\
\vdots & & \vdots \\
\mu_{n-1} & \cdots & \mu_{2 n-2}
\end{array}\right|,
$$




$$
\widetilde{H}_{n}=\left|\begin{array}{cccc}
\mu_{0} & \cdots & \mu_{n-2} & \mu_{n} \\
\vdots & & \vdots & \vdots \\
\mu_{n-1} & \cdots & \mu_{2 n-3} & \mu_{2 n-1}
\end{array}\right|,
$$

where $H_{0}=1$ and $\widetilde{H}_{0}=0$, and $h_{0}(x)=$ const $>0$ is defined by normalization which is chosen for system $\left.\left\{h_{n}(x)\right\}\right|_{n=0} ^{\infty}$ under consideration. The clear view of $h_{n}(x)$ can be found, for instance, in [79] for few lowest orders $n$ at normalization $h_{0}(x)=1$. The specific cases $\alpha=1$ and $\alpha=2$ correspond to Cauchy and Gauss distributions, respectively, which are mostly used in the correlation femtoscopy. For the first case the Laguerre polynomials, $L_{n}(x)$, are used as $\left.\left\{h_{n}(x)\right\}\right|_{n=0} ^{\infty}$; the Hermite polynomials, $H_{n}(x)$, are chosen as the closed system of orthogonal polynomials for the second specific case [80].

The generalized parametrization of (11a) and (11b) contains the important physical information with regard to the possible high irregular geometry of emission region and dynamics of its creation and it is additional with respect to information derived for sets $\mathscr{G}_{1,2}$ of space-time parameters based on traditional Gaussian parametrization. At present there are a few studies [81-84] which used the formalism outlined above for specific case of Gaussian distribution only. But these investigations confirm already the importance of detailed analysis of peak shape of CF. Thus high statistics and parameters of multiparticle final state for FCC energies allow us the qualitative study of complex geometry of emission region for secondary pions.

\section{Summary}

The following conclusions can be obtained by summarizing the results of the present study.

Energy dependence is investigated for main BEC parameters from the set $\mathscr{G}_{1}$ derived in the framework of $3 \mathrm{D}$ Gaussian approach in $p+p$ collisions as well as for the set of important additional observables $\mathscr{G}_{2}$. Analytic function is suggested for approximation of energy dependence of main BEC parameters. The fits demonstrate statistically acceptable qualities for $\lambda$ and for most radii even for taking into account statistical errors of experimental points. Smooth curves calculated for energy dependence of the set $\mathscr{G}_{2}$ of additional BEC parameters agree with corresponding experimental data at least of qualitative level. The estimation of emission duration of pions in $p+p$ collisions increases from about $0.4 \mathrm{fm} / c$ at RHIC energy up to $1.3 \mathrm{fm} / c$ at the LHC energy. Estimations are obtained for wide set of space-time characteristics of pion source at FCC energies on the basis of the fit results for $p+p$ and nucleus-nucleus collisions. The pion source is characterized by linear sizes about $2 \mathrm{fm}$ in $p+p$, about $4-5 \mathrm{fm}$ in $p+\mathrm{Pb}$, and 6-9 fm in $\mathrm{Pb}+\mathrm{Pb}$ collisions at FCC energies. Volume of pion source at freeze-out is estimated from few hundreds of $\mathrm{fm}^{3}$ in $p+p$ through few thousands of $\mathrm{fm}^{3}$ in $p+\mathrm{Pb}$ up to $10^{4} \mathrm{fm}^{3}$ in $\mathrm{Pb}+\mathrm{Pb}$ collisions at FCC energies.

The charged particle density and its critical value are investigated for high energy $p+p$ and $\mathrm{A}+\mathrm{A}$ collisions. The experimental dependence $n_{\mathrm{ch}}^{\mathrm{pp}}\left(\sqrt{s_{\mathrm{pp}}}\right)$ is almost flat and it is described by smooth curves reasonably for various parameterizations of total charged particle multiplicity. $n_{\mathrm{ch}}^{c, \mathrm{pP}}\left(\sqrt{s_{\mathrm{pp}}}\right)$ decreases with energy; nevertheless the estimations of critical value are larger significantly than charged particle density up to FCC energy. Therefore one can not expect the BoseEinstein condensation and appropriate effects for secondary pions in $p+p$ collisions at FCC within the present approach. The charged particle density is noticeably larger in heavy ion collisions than that in $p+p$ at similar collision energies. The experimental dependence $n_{\mathrm{ch}}^{\mathrm{AA}}\left(\sqrt{s_{\mathrm{NN}}}\right)$ increases with energy in difference with $p+p$ reactions. Smooth curves calculated for various approaches of total charged particle multiplicity agree with experimental points. The situation is dramatically different in high energy nucleus-nucleus collisions with respect to $p+p$ case. The charged particle density is in the range of estimations of critical parameter within its large uncertainties from the RHIC energy $\sqrt{s_{\mathrm{NN}}}=$ $0.2 \mathrm{TeV}$ up to the LHC $\sqrt{s_{\mathrm{NN}}}=2.76 \mathrm{TeV}$ which is highest for experimentally available $\mathrm{BEC}$ results in nucleus-nucleus collisions. Furthermore there is clear enhancement of values estimated for $n_{\mathrm{ch}}^{\mathrm{AA}}$ over values for critical density $n_{\mathrm{ch}}^{c, \mathrm{AA}}$ at $\sqrt{s_{\mathrm{NN}}} \gtrsim 10 \mathrm{TeV}$ even with taking into account large statistical uncertainty for $n_{\mathrm{ch}}^{c, \mathrm{AA}}\left(\sqrt{s_{\mathrm{NN}}}\right)$. Thus there is possibility for Bose-Einstein condensation and novel effects, in particular, pion laser in nucleus-nucleus collisions at FCC energy. It seems that the theoretical and experimental developments are essential for future progress in this field as well as for more definite conclusions due to improvement of precision for appropriate quantities.

The generalized parametrization for two-particle BEC correlation function is suggested and it takes into account the expansion in closed system of orthogonal polynomials for general case of nonisotropic central-symmetrical LévyFeldheim distribution. Possibly, the view of BEC CF can be useful for detailed study of correlation peak shape at FCC.

\section{Competing Interests}

The author declares that there is no conflict of interests regarding the publication of this paper.

\section{Acknowledgments}

The author is grateful to Professor E. K. G. Sarkisyan for fruitful discussions and helpful comments.

\section{References}

[1] R. M. Weiner, Introduction to Bose-Einstein Correlations and Subatomic Interferometry, John Wiley \& Sons, Chichester, UK, 2000.

[2] R. Hanbury-Brown and R. Q. Twiss, "LXXIV. A new type of interferometer for use in radio astronomy," The London, Edinburgh, and Dublin Philosophical Magazine and Journal of Science, vol. 45, pp. 663-682, 1954.

[3] R. Hanbury Brown and R. Q. Twiss, "A test of a new type of stellar interferometer on Sirius," Nature, vol. 178, no. 4541, pp. 1046-1048, 1956. 
[4] M. Schaumann, "Potential performance for $\mathrm{Pb}-\mathrm{Pb}, p-\mathrm{Pb}$, and $p$ - $p$ collisions in a future circular collider," Physical Review Accelerators and Beams, vol. 18, no. 9, Article ID 091002, 2015.

[5] N. Armesto, A. Dainese, D. d'Enterria et al., "Heavy-ion physics studies for the Future Circular Collider," Nuclear Physics A, vol. 931, pp. 1163-1168, 2014.

[6] A. Dainese, U. A. Wiedemann, N. Armesto et al., "Heavy ions at the future circular collider," https://arxiv.org/abs/1605.01389.

[7] N. Armesto, A. Dainese, D. d'Enterria et al., "Nuclear collisions at the future circular collider," https://arxiv.org/abs/1601.02963.

[8] N.-B. Chang, S. Cao, B.-Y. Chen et al., "Physics perspectives of heavy-ion collisions at very high energy," Science China Physics, Mechanics \& Astronomy, vol. 59, no. 2, Article ID 621001, 2016.

[9] Z. Fodor and S. D. Katz, "Lattice determination of the critical point of QCD at finite T and $\mu$," Journal of High Energy Physics, vol. 2002, no. 3, p. 14, 2002.

[10] V. A. Okorokov and E. V. Sandrakova, "Influence of fractal embedding in three-dimensional Euclidean space on wave propogation in electro- chromodynamics," Universal Journal of Physics and Application, vol. 1, pp. 196-199, 2013.

[11] V. A. Okorokov, "Energy dependence of femtoscopy properties of pion source in nuclear collisions," https://arxiv.org/abs/1312 .4269 .

[12] S. E. Vance, T. Csörgó, and D. Kharzeev, "Observation of partial $U_{A}(1)$ restoration from two-pion bose-einstein correlations," Physical Review Letters, vol. 81, no. 11, p. 2205, 1998.

[13] S. Pratt, "Pion lasers from high-energy collisions," Physics Letters B, vol. 301, no. 2-3, pp. 159-164, 1993.

[14] S. Pratt, "Deciphering the CENTAURO puzzle," Physical Review C, vol. 50, no. 1, pp. 469-479, 1994.

[15] V. A. Okorokov, "Azimuthally integrated HBT parameters for charged pions in nucleus-nucleus interactions versus collision energy," Advances in High Energy Physics, vol. 2015, Article ID 790646, 13 pages, 2015.

[16] F. Antinori, W. Beusch, I. J. Bloodworth et al., "Centrality dependence of the expansion dynamics in $\mathrm{Pb}-\mathrm{Pb}$ collisions at $158 \mathrm{~A} \mathrm{GeV}^{-1}$," Journal of Physics G: Nuclear and Particle Physics, vol. 27, no. 11, pp. 2325-2344, 2001.

[17] E. K. G. Sarkisyan, A. N. Mishra, R. Sahoo, and A. S. Sakharov, "Multihadron production dynamics exploring the energy balance in hadronic and nuclear collisions," Physical Review D, vol. 93, no. 5, Article ID 054046, 14 pages, 2016.

[18] I. M. Dremin and J. W. Gary, "Hadron multiplicities," Physics Report, vol. 349, no. 4, pp. 301-393, 2001.

[19] E. Abbas, B. Abelev, J. Adam et al., "Centrality dependence of the pseudorapidity density distribution for charged particles in $\mathrm{Pb}-\mathrm{Pb}$ collisions at $\sqrt{s_{N N}}=2.76 \mathrm{TeV}$,' Physics Letters $B$, vol. 726, no. 4-5, pp. 610-622, 2013.

[20] V. A. Okorokov, "Levy distributions for one-dimensional analysis of the Bose-Einstein correlations," https://arxiv.org/abs/1605 .02927.

[21] Y. Sinyukov, "Boson spectra and correlations in small thermalized systems," in Hot Hadronic Matter: Theory and Experiment, J. Letessier, H. G. Gutbrod, and J. Rafelski, Eds., vol. 346 of NATO ASI Series B, pp. 309-322, Plenum, New York, NY, USA, 1995.

[22] S. V. Akkelin and Yu. M. Sinyukov, "The HBT-interferometry of expanding sources," Physics Letters B, vol. 356, no. 4, pp. 525530, 1995.

[23] S. Pratt, "Pion interferometry of quark-gluon plasma," Physical Review D, vol. 33, no. 5, pp. 1314-1327, 1986.
[24] G. F. Bertsch, M. Gong, and M. Tohyama, "Pion interferometry in ultrarelativistic heavy-ion collisions," Physical Review C, vol. 37, no. 5, pp. 1896-1900, 1988.

[25] U. A. Wiedemann and U. Heinz, "Particle interferometry for relativistic heavy-ion collisions," Physics Reports, vol. 319, no. 45, pp. 145-230, 1999.

[26] V. A. Okorokov and E. V. Sandrakova, Fractals in Fundamental Physics. Fractal Properties of Multiparticle Production and Topology of Sample, MEPhI, Moscow, Russia, 2009.

[27] L. Valentin, Subatomic Physics: Nuclei and Particles, Ermann, Paris, France, 1982.

[28] K. N. Mukhin, Experimental Nuclear Physics. V. I., Energoatomizdat, Moscow, Russia, 1983.

[29] V. A. Okorokov, "Energy dependence of space-time extent of pion source in nuclear collisions," in Proceedings of the Workshop on Particle Correlations and Femtoscopy (WPCF '14), T. Csörgó, M. Csanád, and T. Novák, Eds., eConf C140825.8, Scientific Publishing Services at SLAC, Gyongyos, Hungary, August 2014.

[30] G. Alexander and V. Okorokov, "The $\lambda$ energy dependence deduced from Bose-Einstein correlations of $\pi \pi$ pairs produced in pp collisions," Journal of Physics: Conference Series, vol. 675, no. 2, Article ID 022001, 2016.

[31] J. Adam, D. Adamova, M. M. Aggarwal et al., "Two-pion femtoscopy in $p-\mathrm{Pb}$ collisions at $\sqrt{s_{N N}}=5.02 \mathrm{TeV}$," Physical Review C, vol. 91, no. 3, Article ID 034906, 2015.

[32] M. K. Köhler, "Femtoscopic measurements in $p+\mathrm{Pb}$ collisions at $\sqrt{s_{\mathrm{NN}}}=5.02 \sim \mathrm{TeV}$ with ATLAS at the LHC, https://arxiv.org/abs/1601.05632.

[33] E. V. Shuryak, The QCD Vacuum, Hadrons and Superdense Matter, World Scientific Publishing, Singapore, 2004.

[34] E. K. G. Sarkisyan and A. S. Sakharov, "On similarities of bulk observables in nuclear and particle collisions," CERN-PH-TH2004-213, https://arxiv.org/abs/hep-ph/0410324.

[35] E. K. G. Sarkisyan and A. S. Sakharov, "Multihadron production features in different reactions," AIP Conference Proceedings, vol. 828, pp. 35-41, 2006.

[36] E. K. G. Sarkisyan and A. S. Sakharov, "Relating multihadron production in hadronic and nuclear collisions," European Physical Journal C, vol. 70, no. 3, pp. 533-541, 2010.

[37] B. I. Abelev, M. M. Aggarwal, Z. Ahammed et al., "Systematic measurements of identified particle spectra in $p p, d+A u$, and $\mathrm{Au}+\mathrm{Au}$ collisions at the STAR detector," Physical Review C, vol. 79, no. 3, Article ID 034909, 58 pages, 2009.

[38] L. J. Gutay, A. S. Hirsch, R. P. Scharenberg, B. K. Srivastava, and C. Pajares, "De-confinement in small systems: clustering of color sources in high multiplicity $\bar{p} p$ collisions at $\sqrt{s}=1.8 \mathrm{TeV}$," International Journal of Modern Physics E, vol. 24, no. 12, Article ID 1550101, 2015.

[39] J. Cleymans, H. Oeschler, K. Redlich, and S. Wheaton, "Status of chemical freeze-out," Journal of Physics G: Nuclear and Particle Physics, vol. 32, no. 12, pp. S165-S169, 2006.

[40] J. Cleymans, H. Oeschler, K. Redlich, and S. Wheaton, "Comparison of chemical freeze-out criteria in heavy-ion collisions," Physical Review C, vol. 73, no. 3, Article ID 034905, 10 pages, 2006.

[41] W. Li, "Observation of a ridge correlation structure in high multiplicity proton-proton collisions: a brief review," Modern Physics Letters A, vol. 27, no. 19, Article ID 1230018, 2012.

[42] P. Bozek, "Collective flow in $p-\mathrm{Pb}$ and $d-\mathrm{Pb}$ collisions at $\mathrm{TeV}$ energies," Physical Review C, vol. 85, no. 1, Article ID 014911, 2012. 
[43] P. Bozek and W. Broniowski, "Correlations from hydrodynamic flow in $\mathrm{pPb}$ collisions," Physics Letters, Section B: Nuclear, Elementary Particle and High-Energy Physics, vol. 718, no. 4-5, pp. 1557-1561, 2013.

[44] J. D. Bjorken, S. J. Brodsky, and A. S. Goldhaber, "Possible multiparticle ridge-like correlations in very high multiplicity proton-proton collisions," Physics Letters B, vol. 726, no. 1-3, pp. 344-346, 2013.

[45] K. Dusling and R. Venugopalan, "Evidence for BFKL and saturation dynamics from dihadron spectra at the LHC," Physical Review D, vol. 87, no. 5, Article ID 051502, 7 pages, 2013.

[46] K. Dusling and R. Venugopalan, "Explanation of systematics of CMS $\mathrm{p}+\mathrm{Pb}$ high multiplicity dihadron data at $\sqrt{s}_{N N}=$ 5.02 TeV," Physical Review D, vol. 87, no. 5, Article ID 054014, 12 pages, 2013.

[47] B. Schenke and R. Venugopalan, "Eccentric protons? sensitivity of flow to system size and shape in $p+p, p+\mathrm{Pb}$ and $\mathrm{Pb}+\mathrm{Pb}$ collisions," Physical Review Letters, vol. 113, no. 10, Article ID 102301, 2014.

[48] K. Dusling, W. Li, and B. Schenke, "Novel collective phenomena in high-energy proton-proton and proton-nucleus collisions," International Journal of Modern Physics E, vol. 25, no. 1, Article ID 1630002, 2016.

[49] V. Khachatryan, A. M. Sirunyan, A. Tumasyan et al., "Observation of long-range, near-side angular correlations in protonproton collisions at the LHC," Journal of High Energy Physics, vol. 2010, article 91, 2010.

[50] G. Aad, B. Abbott, J. Abdallah et al., "Observation of long-range elliptic azimuthal anisotropies in $\sqrt{s}=13$ and $2.76 \mathrm{TeV} p p$ collisions with the ATLAS detector," Physical Review Letters, vol. 116, no. 17, Article ID 172301, 2016.

[51] V. Khachatryan, A. M. Sirunyan, A. Tumasyan et al., "Measurement of long-range near-side two-particle angular correlations in $p p$ collisions at $\sqrt{s}=13 \mathrm{TeV}$," Physical Review Letters, vol. 116, no. 17, Article ID 172302, 2016.

[52] M. M. Aggarwal, Z. Ahammed, A. V. Alakhverdyants et al., "Pion femtoscopy in $p+p$ collisions at $\sqrt{s}=200 \mathrm{GeV}$," Physical Review C, vol. 83, Article ID 064905, 2011.

[53] K. Aamodt, T. Antičić, S. Gotovac et al., "Femtoscopy of $p p$ collisions at $\sqrt{s}=0.09$ and $7 \mathrm{TeV}$ at the LHC with two-pion Bose-Einstein correlations," Physical Review D, vol. 84, no. 11, Article ID 112004, 2011.

[54] K. Aamodt, N. Abel, U. Abeysekara et al., "Charged-particle multiplicity measurement in proton-proton collisions at $\sqrt{s}=$ 0.9 and $2.36 \mathrm{TeV}$ with ALICE at LHC," The European Physical Journal C, vol. 68, no. 1, pp. 89-108, 2010.

[55] J. Adam, D. Adamova, M. Aggarwal et al., "Charged-particle multiplicities in proton-proton collisions at $\sqrt{s}=0.9$ to $8 \mathrm{TeV}$," https://arxiv.org/abs/1509.07541.

[56] J. F. Grosse-Oetringhaus and K. Reygers, "Charged-particle multiplicity in proton-proton collisions," Journal of Physics G: Nuclear and Particle Physics, vol. 37, no. 8, Article ID 083001, 2010.

[57] A. Heister, S. Schael, R. Barate et al., "Studies of QCD at $\mathrm{e}^{+} \mathrm{e}^{-}$ centre-of-mass energies between 91 and 209 GeV," The European Physical Journal C-Particles and Fields, vol. 35, no. 4, pp. 457486, 2004.

[58] R. Nouicer, B. B. Back, M. D. Baker et al., "Pseudorapidity distributions of charged particles in $\mathrm{d}+\mathrm{Au}$ and $\mathrm{p}+\mathrm{p}$ collisions at $\sqrt{s_{N N}}=200 \mathrm{GeV}$,' Journal of Physics G: Nuclear and Particle Physics, vol. 30, no. 8, p. S1133, 2004.
[59] G. J. Alner, R. E. Ansorge, B. Åsman et al., "Scaling of pseudorapidity distributions at c.m. energies up to $0.9 \mathrm{TeV}$," Zeitschrift für Physik C Particles and Fields, vol. 33, no. 1, pp. 1-6, 1986.

[60] R. E. Ansorge, B. Åsman, L. Burow et al., "Charged particle multiplicity distributions at $200-\mathrm{GeV}$ and $900-\mathrm{GeV}$ center-ofmass energy," Zeitschrift für Physik C, vol. 43, no. 3, pp. 357-374, 1989.

[61] T. Alexopoulos, C. Allen, E. W. Anderson et al., "Study of source size in $p \bar{p}$ collisions at $\sqrt{s}=1.8 \mathrm{TeV}$ using pion interferometry," Physical Review D, vol. 48, no. 5, p. 1931, 1993.

[62] L. Lovás, The Bose-Einstein correlations [Ph.D. thesis], 2008.

[63] V. Khachatryan, A. M. Sirunyan, A. Tumasyan et al., "First measurement of bose-einstein correlations in proton-proton Collisions at $\sqrt{s}=0.9$ and $2.36 \mathrm{TeV}$ at the LHC," Physical Review Letters, vol. 105, no. 3, Article ID 032001, 2010.

[64] T. Csörgó and J. Zimányi, "Analytic solution of the pion-laser model," Physical Review Letters, vol. 80, no. 5, p. 916, 1998.

[65] T. Csörgó, "Coherent states of the creation operator from fully developed Bose-Einstein condensates," Heavy Ion Phys, vol. 9, p. 161, 1999.

[66] J. Zimányi and T. Csörgó, "Multi-boson correlations using wave-packets I: exact results," Acta Physica Hungarica New Series Heavy Ion Physics, vol. 9, no. 2-3, pp. 241-281, 1999.

[67] S. V. Akkelin and Yu. M. Sinyukov, "DCC decay as possible mechanism of 'pion laser' radiation," Nuclear Physics A, vol. 661, no. 1-4, pp. 613c-616c, 1999.

[68] W. Q. Chao, C. S. Gao, and Q. H. Zhang, "Multi-pion BoseEinstein correlation effects on two-pion interferometry," Journal of Physics G: Nuclear and Particle Physics, vol. 21, no. 6, pp. 847859, 1995.

[69] T. Csörgő, S. Hegyi, and W. A. Zajc, "Bose-Einstein correlations for Lévy stable source distributions," The European Physical Journal C, vol. 36, no. 1, pp. 67-78, 2004.

[70] V. Feller, An Inroduction to Probability Theory and it's Applications, John Wiley \& Sons, New York, NY, USA, 1975.

[71] V. M. Zolotarev, One-Dimensional Stable Distributions, Nauka, Moscow, Russia, 1983.

[72] G. Samorodnitzky and M. S. Taqqu, Stable Non-Gaussian Random Processes, Chapman \& Hall/CRC, New York, NY, USA, 1994.

[73] V. V. Uchaikin, "Anomalous diffusion and fractional stable distributions," Journal of Experimental and Theoretical Physics, vol. 97, no. 4, pp. 810-825, 2003.

[74] H. B. Stahl and V. Totik, General Orthogonal Polynomials, Cambridge University Press, Cambridge, UK, 1992.

[75] A. P. Magnus, "Preud's equations for orthogonal polynomials as discrete Painlevé equations," https://arxiv.org/abs/math/ 9611218.

[76] E. Levin and D. S. Lubinsky, Orthogonal Polynomials for Exponential Weights, Springer, New York, NY, USA, 2001.

[77] T. Kasuda and R. Sakai, "Orthonormal polynomials with generalized Freud-type weights," Journal of Approximation Theory, vol. 121, no. 1, pp. 13-53, 2003.

[78] A. P. Prudnikov, Y. A. Brichkov, and O. E. Marichev, Integrals and Serieses. Elementary Functions, Nauka, Moscow, Russia, 1981.

[79] T. Novák, T. Csörgő, H. C. Eggers, and M. de Kock, "Model independent analysis of nearly Lévy correlations," https://arxiv.org/ abs/1604.05513. 
[80] T. Csörgö and S. Hegyi, "Model independent shape analysis of correlations in 1, 2 or 3 dimensions," Physics Letters B, vol. 489, no. 1-2, pp. 15-23, 2000.

[81] J. Adams, M. M. Aggarwal, Z. Ahammed et al., "Pion interferometry in Au+Au collisions at $\sqrt{s_{N N}}=200 \mathrm{GeV}$," Physical Review C, vol. 71, no. 4, Article ID 044906, 2005.

[82] H. C. Eggers, B. Buschbeck, and F. J. October, "Multidimensional HBT correlations in proton-antiproton collisions at $\operatorname{root}(\mathrm{s})=630 \mathrm{GeV}$," https://arxiv.org/abs/hep-ex/0511050.

[83] B. Abelev, J. Adamak, D. Adamová et al., "Freeze-out radii extracted from three-pion cumulants in $\mathrm{pp}, \mathrm{p}-\mathrm{Pb}$ and $\mathrm{Pb}-\mathrm{Pb}$ collisions at the LHC," Physics Letters B, vol. 739, pp. 139-151, 2014.

[84] B. Abelev, J. Adam, D. Adamová et al., “Two- and three-pion quantum statistics correlations in $\mathrm{Pb}-\mathrm{Pb}$ collisions at $\sqrt{s_{N N}}=$ 2.76 TeV at the CERN large hadron collider," Physical Review C, vol. 89, no. 2, Article ID 024911, 2014. 

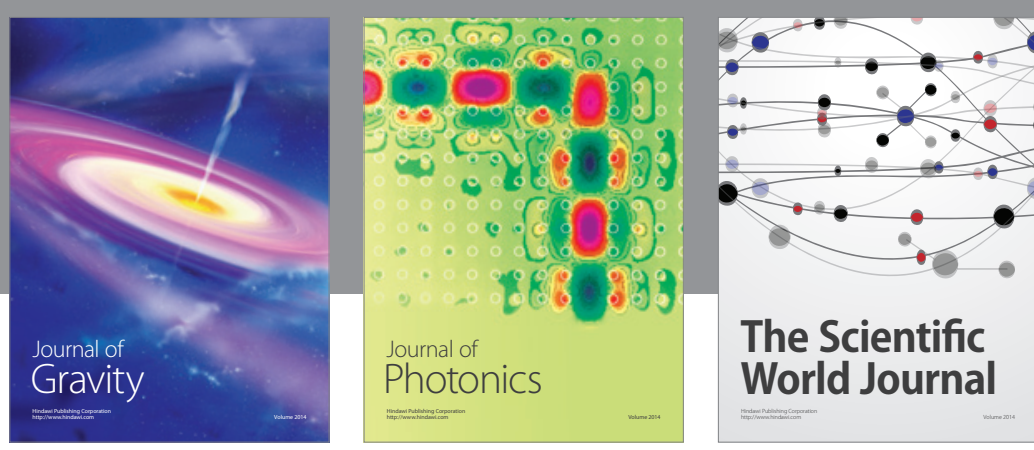

The Scientific World Journal
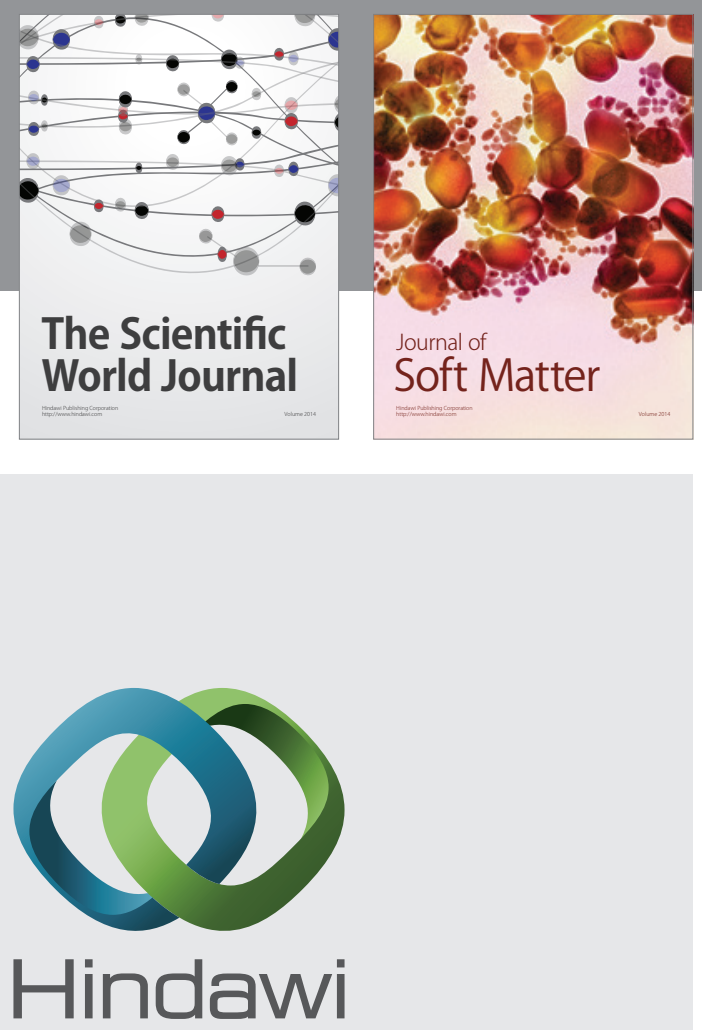

Submit your manuscripts at

http://www.hindawi.com

nternational Journal of

Statistical Mechanics
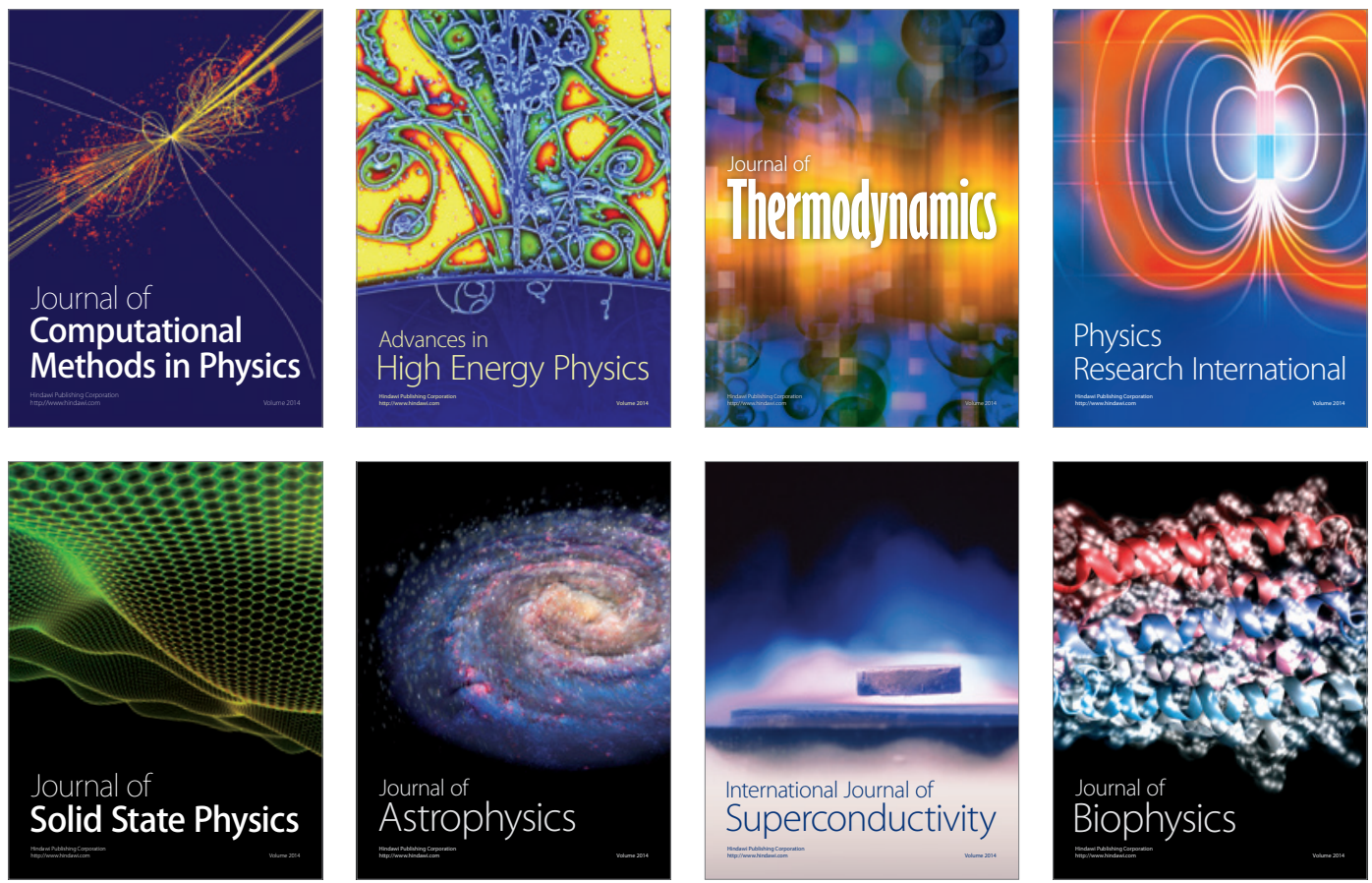
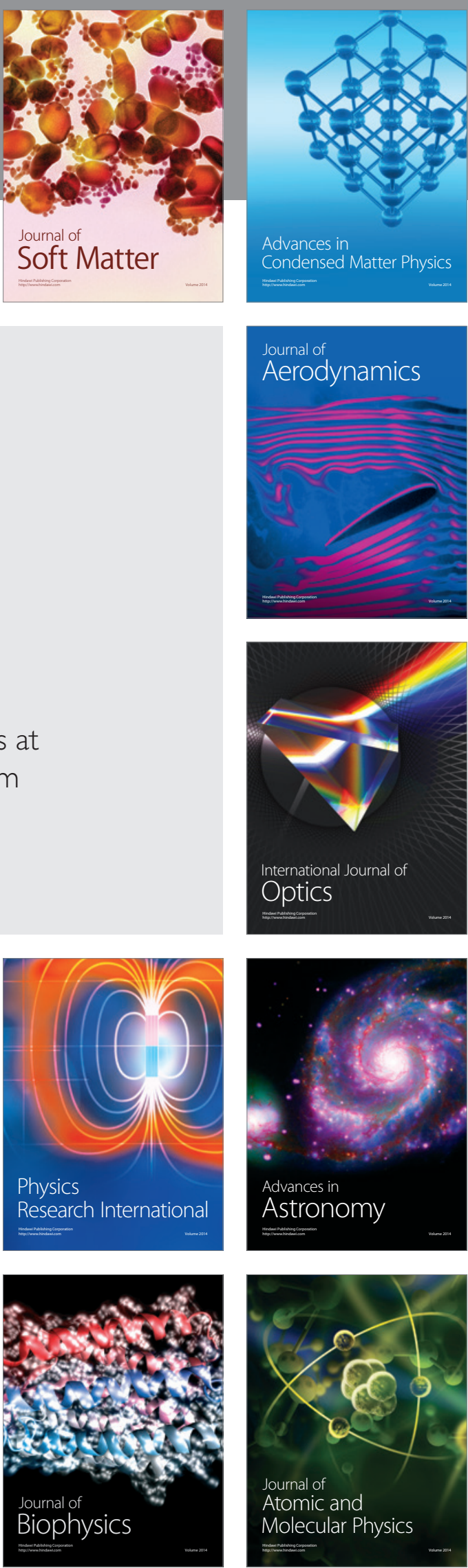